Supporting Information

\title{
Light-Click in-Situ Self-Assembly (LISA) of Superhelical Nanofibers and Their Helicity Hierarchy Control
}

Liang Chen, ${ }^{1,2}$ Xuefeng Li, ${ }^{1}$ and Qiang Yan $^{1,2 *}$

${ }^{1}$ State Key Laboratory of Molecular Engineering of Polymers, Fudan University.

${ }^{2}$ Department of Macromolecular Science, Fudan University, Shanghai 200433, China.

\section{Content}

1. Materials

2. Methods. Scheme S1

3. Synthetic Routes. Scheme S2-S3

4. Supporting Characterization

NMR of Photoligation. Figure S1

MALDI-TOF Mass Spectroscopy of Photoligation. Figure S2

HPLC of Photoligation Figure S3

UV-vis Turbidity for LISA Process. Figure S4

DLS Monitoring for LISA Process. Figure S5

TEM Particle Statistics for Fiber Distribution Figure S6

CLSM for the Fluorescent Superhelical Fibers Figure S7

Photoclick Reaction Kinetic Studies Figure S8

TEM Showing the Initial Morphology. Figure S9

TEM Test for Non-helical Filament Nanostructure. Figure S10

Self-Assembly Fashion of Non-helical Filament. Figure S11

TEM for LISA Phase Evolutionary Process. . . Figure S12

UV-vis Spectra Monitoring LISA Process. Figure S13

CD Spectra Monitoring LISA Process. Figure S14

5. Structural Characterization of All Synthetic Samples. .Figure S15-Figure S25 


\section{Materials.}

4-Formylbenzoic acid (Energy Chemical, 98\%), benzenesulfonohydrazide (TCl, 98\%), 4-(diethylamino)-2-hydroxybenzaldehyde (TCl, 98\%), 3-bromo-1-propanol (Sigma, 97\%), 2-benzothiazoleacetonitrile (Energy Chemical, 98\%), 11-maleimidoundecanoic (Adamas, 97\%), oxalyl chloride (Alfa Aesar, 98\%), trimethylamine (Energy Chemical, 99.5\%, extra dry), aniline (Energy Chemical, 99.5\%), dicyclohexylcarbodiimide (DCC, Energy Chemical, 98\%), 4-dimethylaminopyridine (DMAP, Energy Chemical, 98\%), pyridine (Sigma, 99.5\%, extra dry), 18-crown-6 (Alfa Aesar, 99\%), piperidine (Sigma, 99.5\%), sodium nitrite $\left(\mathrm{NaNO}_{2}\right.$, Energy Chemical, $\left.99 \%\right)$, potassium carbonate $\left(\mathrm{K}_{2} \mathrm{CO}_{3}\right.$, Energy Chemical, 99\%), poly(ethylene oxide) monomethyl ethers $\left(M_{\mathrm{n}} \approx 1000,2000\right.$, 3500 and $5000 \mathrm{~g} / \mathrm{mol}$, Sigma-Aldrich). All of the solvents, including ethanol, acetone tetrahydrofuran (THF), dichloromethane $\left(\mathrm{CH}_{2} \mathrm{Cl}_{2}\right)$, and ethyl acetate, were purchased from Shanghai Chemical Ltd Co. Anhydrous $\mathrm{CH}_{2} \mathrm{Cl}_{2}$ and dimethyl formamide (DMF) were purchased from Sigma and used without purification. Unless specially mentioned, all chemicals were used as received.

\section{Methods.}

Nuclear Magnetic Resonance (NMR) was measured by AVANCE III HD $400 \mathrm{MHz}$ of Bruker BioSpin International for characterization of the ${ }^{1} \mathrm{H}$ NMR and ${ }^{13} \mathrm{C}$ NMR spectra of all the samples and used $\mathrm{CDCl}_{3}$ or $d_{6}$-DMSO as NMR solvent. Matrix assisted laser desorption ionization time-of-flight mass spectroscopy (MALDI-TOF MS) was used for characterization of the molecular weights of all the samples by an AB SCIEX 5800 system. Trans-2-[3-(4-tert-Butylphenyl)-2-methyl-2-propenylidene] (DCTB) was used as the matrix. Gel Permeation Chromatography (GPC) tests were performed on a GPC system. The system is equipped with a Waters degasser, a Waters 515 HPLC pump, a Wyatt Optilab DSP refractive index detector and a Wyatt mini DAWN light scattering detector with the detection wavelength set at $658 \mathrm{~nm}$. The chromatographic columns include one Styragel guard column $(7.5 \times 50 \mathrm{~mm})$, one Styragel HR3 column $(5 \mu \mathrm{m}$, $7.8 \times 300 \mathrm{~mm}$, molecular range $\left.500 \sim 3 \times 10^{4} \mathrm{~g} / \mathrm{mol}\right)$ and one Styragel HR5E column (5 
$\mu \mathrm{m}, 7.8 \times 300 \mathrm{~mm}$, molecular range $2000 \sim 4 \times 10^{6} \mathrm{~g} / \mathrm{mol}$ ). HPLC-grade tetrahydrofuran (THF) was used as mobile phase at a flow rate of $1.0 \mathrm{~mL} / \mathrm{min}$ at $30^{\circ} \mathrm{C}$. The molecular weights and polydispersities were calculated using the Wyatt ASTRA software and the dn/dc values were off-line obtained. Dynamic Light Scattering (DLS) tests used a Malvern Zetasizer Nano ZS to determine the hydrodynamic diameters and distribution of the samples before and after light trigger. The scattering angel is fixed on $90{ }^{\circ} \mathrm{C}$. $\boldsymbol{U}$ vis Spectroscopy (UV-vis) was recorded by an Agilent Cary-60 UV-Vis spectroscopy. The turbidity tests were carried out on a constant wavelength of $600 \mathrm{~nm}$ to monitor the optical transmittance change of two precursor solution during the light irradiation. The UV-vis absorption variation of two precursors during the light irradiation were obtained by measuring the aliquots of solution taken out at given time intervals. Fluorescent Spectroscopy (FS) was performed by a HITACHI F-7100 Fluorescence Spectrometer. The fluorescent emission variation of two precursors during the light irradiation were obtained by measuring the aliquots of solution taken out at given time intervals. Atomic Force Microscope (AFM) were performed on a Bruker Dimension ICON equipped with a J scanner. The specimen was prepared by drop-casting of $\sim 10 \mu \mathrm{L}$ solutions onto the freshly cleaved mica without purification. AFM measurements adopted the PeakForce Tapping mode with a particular SCANAYST-AIR tip (spring constant $40 \mathrm{~N} / \mathrm{m}$, Bruker) and the height, width and pitch of all the assemblies were analyzed by the AFM NanoScope Analyst Software. Transmission Electron Microscopy (TEM) was tested using a JEOL 2000 EX electron microscope operated at $200 \mathrm{kV}$. The specimen was prepared by drop-casting of $10 \mu \mathrm{L}$ solutions onto the carbon-coated copper grid. Then the solution was blotted away and $10 \mu \mathrm{L}$ of $1.0 \mathrm{wt} \%$ uranyl acetate solution was dropped to strain the specimen for about 20 seconds. Scanning Electron Microscope (SEM) was performed on a Zeiss Gemini SEM500 electron microscope. Before observation the surface of silicon substrates were sputtered with a thin layer of $\mathrm{Au}$ for increasing the electrical conductivity. Circular Dichroism (CD) was recorded on a JASCO J-175 spectrometer. The CD spectra were recorded from the two precursor solution $(5.0 \mathrm{wt} \%)$ during the light irradiation by measuring the aliquots of solution taken out at given time intervals. 
Light Irradiation Experiments. All the light irradiation tests of sample solutions were carried out in a customized black box. The black box is equipped with a magnetic stirrer on the bottom, a circular-shaped lamp frame with three fluorescent lamps $(313 \mathrm{~nm}, 8$ $\mathrm{W}$ of each lamp), and three legged holder with reactive tubes in the middle, as shown in the Scheme S1. Before the photoclick reaction, the two molecular precursors $\left(\mathbf{E}_{n} \mathbf{A z}\right.$ and MCV $, n=23,45,80$ and 113) were mixed in the reactive tube in a given molar ratio and then undergo three freeze-vacuum-thaw cycles to remove the oxygen in the tubes. The light irradiation can be on-demand turned on and off by external switch to realize the ON/OFF assembly. During the photoreaction, aliquots of solutions $(20 \mu \mathrm{L})$ from the stock solution were taken out at a given time intervals for further UV-vis, FS and CD measurements and TEM, AFM and SEM microscopic observation.

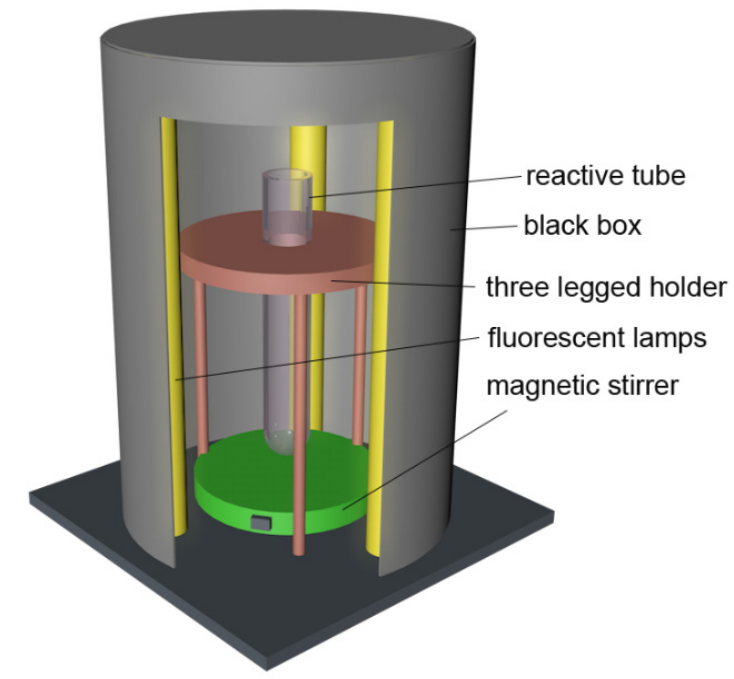

Scheme S1. Schematic representation of the photoreaction equipment.

Light-Click In-Situ Self-Assembly (LISA) Process. $0.1 \mathrm{~mL}$ of a $35 \mathrm{mM} \mathbf{E}_{45} \mathrm{AZ}$ solution in THF, $0.1 \mathrm{~mL}$ of a $35 \mathrm{mM}$ MCV solution in THF were dropwise added to a Schlenk tube with $1.4 \mathrm{~mL}$ of THF and $0.4 \mathrm{~mL}$ of water. The final total concentration of the two reactive molecular precursors was $\sim 5 \mathrm{wt} \%$ and the ratio of $\mathrm{THF} /$ water is $4 / 1$ in volume. After three freeze-vacuum-thaw cycles to remove oxygen, the binary mixture solution was then subjected to $313 \mathrm{~nm}$ UV irradiation for one hour. During the irradiation, the two precursors can in-situ self-assembly to form diversiform nanostructures. 


\section{Synthetic Routes.}

\subsection{Synthesis of the Reactive Precursor, Tetrazole-Capped Poly(ethylene oxide)}

$\left(\mathrm{E}_{n} \mathrm{Az}, n=23,45,80\right.$, and 113)
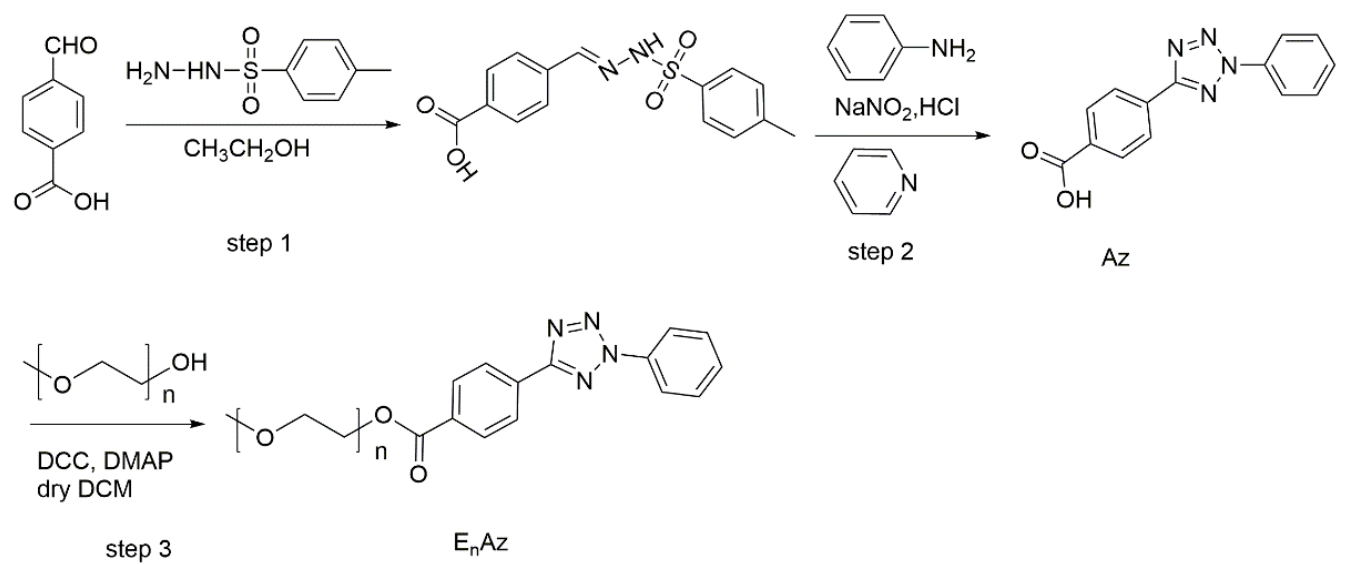

Scheme S2. Synthetic route of the molecular precursor $\left(\mathbf{E}_{n} \mathbf{A z}\right)$.

Synthesis of (E)-4-((2-tosylhydrazineylidene)methyl)benzoic acid. The typical process was performed according to a literature procedure. ${ }^{1}$ 4-Formylbenzoic acid (3.02 g, 20 mmol) and benzenesulfonohydrazide $(3.44 \mathrm{~g}, 100 \mathrm{mmol})$ were together added into a round-bottom flask dissolved in $200 \mathrm{~mL}$ of ethanol. After stirring for $30 \mathrm{~min}$ at room temperature, the reaction was stopped and $400 \mathrm{~mL}$ of water was added into the mixture to obtain a white precipitation. The formed precipitation was filtered off and dried in vacuum to obtain crystalline solids (4.96 g, yield: $78 \%) .{ }^{1} \mathrm{H}$ NMR $(d 6-\mathrm{DMSO}, \delta, \mathrm{ppm})$ : 13.07 (s, 1H), 11.67 (s, 1H), 7.96 (d, 2H), $7.92(\mathrm{~s}, 1 \mathrm{H}), 7.76$ (d, 2H), 7.67 (d, 2H), 7.42 $(\mathrm{d}, 2 \mathrm{H}), 2.36(\mathrm{~s}, 3 \mathrm{H})$.

Synthesis of tetrazole-derived benzoic acid (Az). A typical synthetic method was based on the literature. ${ }^{2}$ The resulting (E)-4-((2-tosylhydrazineylidene)methyl)benzoic acid (4.92 g, $20 \mathrm{mmol}$ ) was dissolved in $120 \mathrm{~mL}$ of pyridine to give solution A. Under icewater bath, a solution of $\mathrm{NaNO}_{2}(1.38 \mathrm{~g}, 20 \mathrm{mmol})$ in $8 \mathrm{~mL}$ water was added dropwise to a cooled mixture of aniline $(1.86 \mathrm{~g}, 20 \mathrm{mmol})$ dissolved in $32 \mathrm{~mL}$ of water-ethanol (1:1) and $5.2 \mathrm{~mL}$ of concentrated $\mathrm{HCl}$ to give solution B. Solution B was added slowly to solution $\mathrm{A}$ at $0{ }^{\circ} \mathrm{C}$ and the reaction mixture was then extracted with ethyl acetate (200 
$\mathrm{mL})$ for three times. Precipitates can be formed after adding $1 \mathrm{~L}$ of $\mathrm{HCl}(3 \mathrm{M})$ to the combined organic layers, and was collected and dried to afford the compound (2.94 g, yield: $71 \%) .{ }^{1} \mathrm{H}$ NMR (d6-DMSO, $\left.\delta, \mathrm{ppm}\right): 13.27(\mathrm{~s}, 1 \mathrm{H}), 8.30(\mathrm{~d}, 2 \mathrm{H}), 8.18(\mathrm{t}, 4 \mathrm{H})$, $7.72(\mathrm{t}, 2 \mathrm{H}), 7.65(\mathrm{t}, 1 \mathrm{H})$.

Synthesis of tetrazole-capped poly(ethylene oxide) $\left(\boldsymbol{E}_{\boldsymbol{n}} \boldsymbol{A} \boldsymbol{z}\right)$. As a typical procedure, the above tetrazole compound $\mathrm{Az}(0.29 \mathrm{~g}, 1.1 \mathrm{mmol})$, poly(ethylene glycol) monomethyl ether $\left(M_{\mathrm{n}} \approx 2000 \mathrm{~g} / \mathrm{mol}, 0.58 \mathrm{~g}, 0.29 \mathrm{mmol}\right)$, and DMAP (27 mg, $\left.0.22 \mathrm{mmol}\right)$ were dissolved in a mixture of dry DCM $(5 \mathrm{~mL})$ and DMF $(0.5 \mathrm{~mL})$. DCC $(0.12 \mathrm{~g}, 0.58$ mmol) was subsequently added at $0{ }^{\circ} \mathrm{C}$. The reaction was then reacted at room temperature for 24 hours. The byproduct urea precipitation was filtered and the solvent was removed under reduced pressure. The target product was obtained by re-dissolution in THF followed by precipitation in diethyl ether $(0.36 \mathrm{~g}$, yield: $55 \%)$. ${ }^{1} \mathrm{H}$ NMR $\left(d_{6}-\right.$ DMSO, $\delta, \mathrm{ppm}): 8.34(\mathrm{t}, 2 \mathrm{H}), 8.21(\mathrm{~m}, 4 \mathrm{H}), 7.72(\mathrm{t}, 2 \mathrm{H}), 7.65(\mathrm{t}, 1 \mathrm{H}), 4.45(\mathrm{t}, 2 \mathrm{H}), 3.79$ (t, 2H), 3.53-3.44 (m, 158H), 3.24 (s, 3H). MALDI-TOF MS (m/z): For E Es Az $\left[\mathrm{M}+\mathrm{K}^{+}\right]$, calcd. 2081.37; found. 2081.17. GPC: $M_{\mathrm{n}}=2240 \mathrm{~g} / \mathrm{mol}, \emptyset=1.02$.

\subsection{Synthesis of the Reactive Precursor, Maleimide-Ended Cyanovinylene (MCV).}
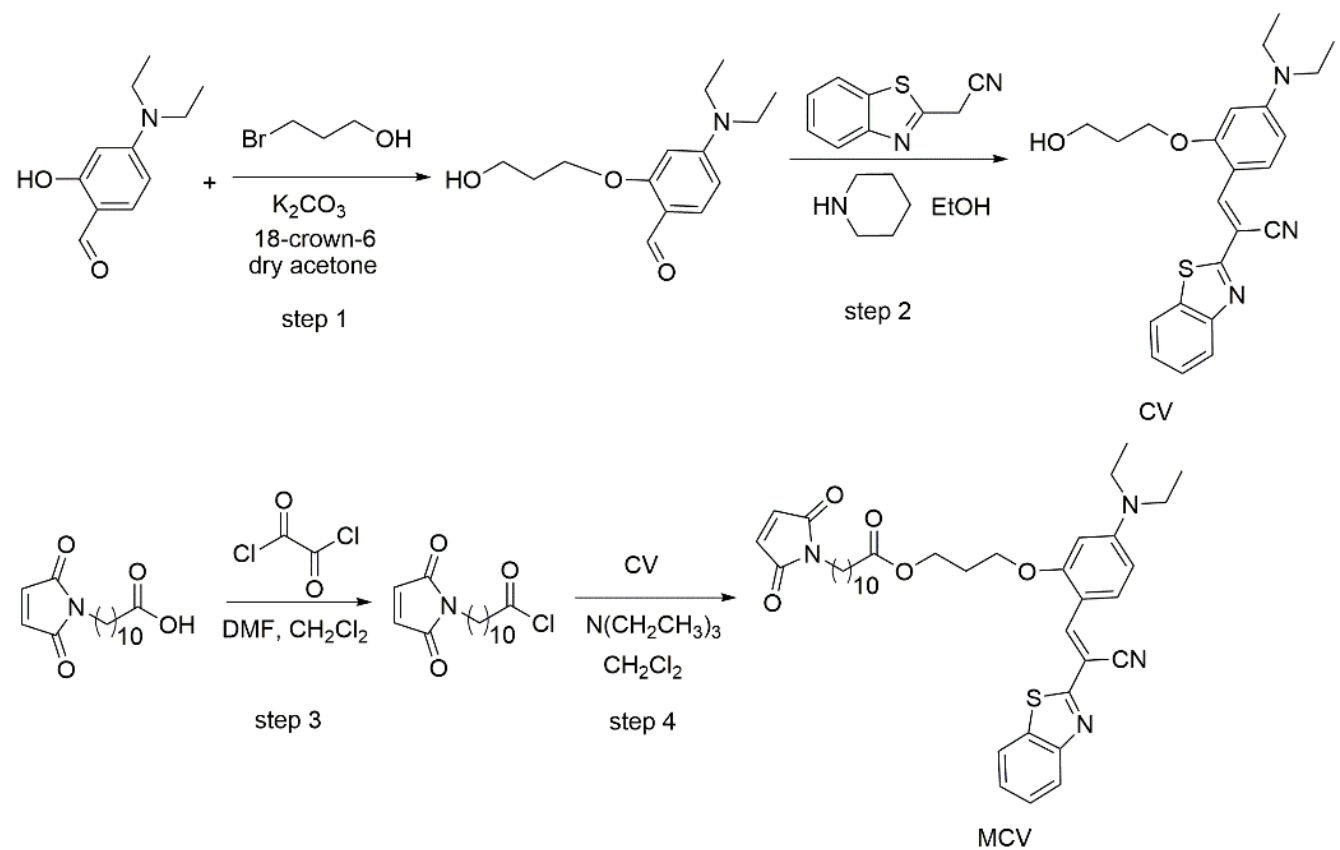

Scheme S3. Synthetic route of the molecular precursor (MCV). 
Synthesis of 4-(diethylamino)-2-(3-hydroxypropoxy)benzaldehyde. The synthetic way was according to the literature. ${ }^{3}$ 4-(Diethylamino)-2-hydroxybenzaldehyde (3.86 g, 20 mmol, 1.0 equiv.) and 3-bromo-1-propanol (2.83 g, $20.5 \mathrm{mmol}, 1.02$ equiv.) were dissolved in $2 \mathrm{~mL}$ of anhydrous acetone. Then potassium carbonate $(4.14 \mathrm{~g}, 30 \mathrm{mmol}$, 1.5 equiv.) and 18 -crown-6 ( $80 \mathrm{mg}, 0.03 \mathrm{mmol}, 0.015$ equiv.) were added into the above solution. The mixture was reacted overnight through reflux. After filtration, the solvent was removed and the residue was re-dissolved in $160 \mathrm{~mL}$ dichloromethane/water (1:1 $\mathrm{v} / \mathrm{v})$. The aqueous layer was extracted for three times with dichloromethane $(100 \mathrm{~mL})$ and the combined organic layers were dried by anhydrous sodium sulfate. The solvent was removed under reduced pressure, and then recrystallize two times using petroleum ether/ethyl acetate $(2 / 1, \mathrm{v} / \mathrm{v})$. The purified solid was obtained after filtration and drying under reduced pressure (1.47 g, yield: 29\%). ${ }^{1} \mathrm{H}$ NMR $\left(\mathrm{CDCl}_{3}, \delta, \mathrm{ppm}\right): 9.94(\mathrm{~s}, 1 \mathrm{H})$, $7.58(\mathrm{~d}, 1 \mathrm{H}), 6.28(\mathrm{~d}, 1 \mathrm{H}), 6.06(\mathrm{~d}, 1 \mathrm{H}), 4.45(\mathrm{t}, 1 \mathrm{H}), 4.18(\mathrm{t}, 2 \mathrm{H}), 3.88(\mathrm{t}, 2 \mathrm{H}), 3.41(\mathrm{~m}$, 4H), $2.10(\mathrm{~m}, 2 \mathrm{H}), 1.21(\mathrm{t}, 6 \mathrm{H})$. MALDI-TOF MS $(\mathrm{m} / \mathrm{z})$ : For $\mathrm{C}_{14} \mathrm{H}_{21} \mathrm{NO}_{3}\left[\mathrm{M}+\mathrm{H}^{+}\right]$, calcd. 252.33; found. 252.27 .

Synthesis of cyanovinylene fluorophore $(\mathrm{CV})$. The synthetic procedure of CV was based on the literature. ${ }^{4}$ The resulting 4-(diethylamino)-2-(3-hydroxypropoxy)benzaldehyde (1.47 g, 5.85 mmol, 2 equiv.) and 2-benzothiazoleacetonitrile (1.07 g, 6.14 mmol, 2.1 equvi.) were dissolved in $90 \mathrm{~mL}$ of ethanol at room temperature. Then piperidine $(6.2$ $\mathrm{mL}, 58.5 \mathrm{mmol}, 21$ equvi.) was added to the above solution. The reaction mixture was reacted at room temperature with stirring overnight and the resulted precipitation was collected by filtration, washed with cold ethanol. The crude solid was recrystallized by ethanol to afford $\mathrm{CV}$ as an orange solid (1.64 g, yield: $68.9 \%) .{ }^{1} \mathrm{H} \mathrm{NMR}\left(\mathrm{CDCl}_{3}, \delta\right.$, ppm): $8.55(\mathrm{~s}, 1 \mathrm{H}), 8.41(\mathrm{~d}, 1 \mathrm{H}), 8.01(\mathrm{~d}, 1 \mathrm{H}), 7.82(\mathrm{~d}, 1 \mathrm{H}), 7.46(\mathrm{t}, 1 \mathrm{H}), 7.34(\mathrm{t}, 1 \mathrm{H})$, $6.42(\mathrm{~d}, 1 \mathrm{H}), 6.18(\mathrm{~s}, 1 \mathrm{H}), 4.21(\mathrm{t}, 2 \mathrm{H}), 4.00(\mathrm{t}, 2 \mathrm{H}), 3.44(\mathrm{~m}, 4 \mathrm{H}), 2.16(\mathrm{~m}, 2 \mathrm{H}), 1.24$ (t, 6H). MALDI-TOF MS (m/z): For $\mathrm{C}_{23} \mathrm{H}_{25} \mathrm{~N}_{3} \mathrm{O}_{2} \mathrm{~S}\left[\mathrm{M}+\mathrm{Na}^{+}\right]$, calcd. 430.17; found. 430.40 . 
Synthesis of Alkyl Maleimide-derived Cyanovinylene (MCV). The typical synthesis was performed according to the literature procedure. ${ }^{5,6}$ 11-Maleimidoundecanoic (11-MUA; $0.560 \mathrm{~g}, 2 \mathrm{mmol}, 1.0$ equiv.) was dissolved in anhydrous $\mathrm{CH}_{2} \mathrm{Cl}_{2}(10 \mathrm{~mL})$ and DMF (10 $\mathrm{mL}$ ) and cooled to $0{ }^{\circ} \mathrm{C}$. Oxalyl chloride (756 $\mu \mathrm{L}, 4 \mathrm{mmol}, 2.0$ equiv.) was subsequently added dropwise by syringe and the mixture was stirred at room temperature for 3 hours. Then, the reaction mixture was evaporated to afford 11-maleimidoundecanoyl chloride (11-MUC) as a clear oil, which is used without any further purification. For further reaction, the above resulted $\mathrm{CV}(0.750 \mathrm{~g}, 1.8 \mathrm{mmol}, 0.9$ equiv. $)$ and triethylamine $(0.24$ g, 2.4 mmol, 1.2 equiv.) were dissolved in $35 \mathrm{~mL}$ of anhydrous $\mathrm{CH}_{2} \mathrm{Cl}_{2}$ and cooled to $0{ }^{\circ} \mathrm{C} .11-\mathrm{MUC}$ was subsequently added dropwise by syringe and the mixture was stirred at room temperature overnight. After filtration, the solution was extracted three times with $\mathrm{NaOH}$ solution $(0.1 \mathrm{M})$ and the organic layers were dried over anhydrous sodium sulfate. After removal of the solvent under reduced pressure, the crude product was purified by chromatography (silica gel, petroleum ether/ethyl acetate $2: 1 \mathrm{v} / \mathrm{v}, 0.51 \mathrm{~g}$, yield: $48 \%) .{ }^{1} \mathrm{H}$ NMR $\left(\mathrm{CDCl}_{3}, \delta, \mathrm{ppm}\right): 8.54$ (s, 1H), 8.45 (d, 1H), 8.06 (d, 1H), 7.85 (d, 1H), $7.49(\mathrm{~m}, 1 \mathrm{H}), 7.38(\mathrm{~m}, 1 \mathrm{H}), 6.67(\mathrm{~s}, 2 \mathrm{H}), 6.57(\mathrm{~d}, 1 \mathrm{H}), 4.36(\mathrm{t}, 2 \mathrm{H}), 4.19(\mathrm{t}$, 2H), $3.49(\mathrm{~m}, 6 \mathrm{H}), 2.31(\mathrm{~m}, 2 \mathrm{H}), 1.28 \sim 1.57(\mathrm{~m}, 24 \mathrm{H})$. MALDI-TOF MS $(\mathrm{m} / \mathrm{z})$ : For $\mathrm{C}_{38} \mathrm{H}_{46} \mathrm{~N}_{4} \mathrm{O}_{5} \mathrm{~S}\left[\mathrm{M}+\mathrm{Na}^{+}\right.$], calcd. 693.32; found. 693.76. 


\section{Supporting Characterization.}

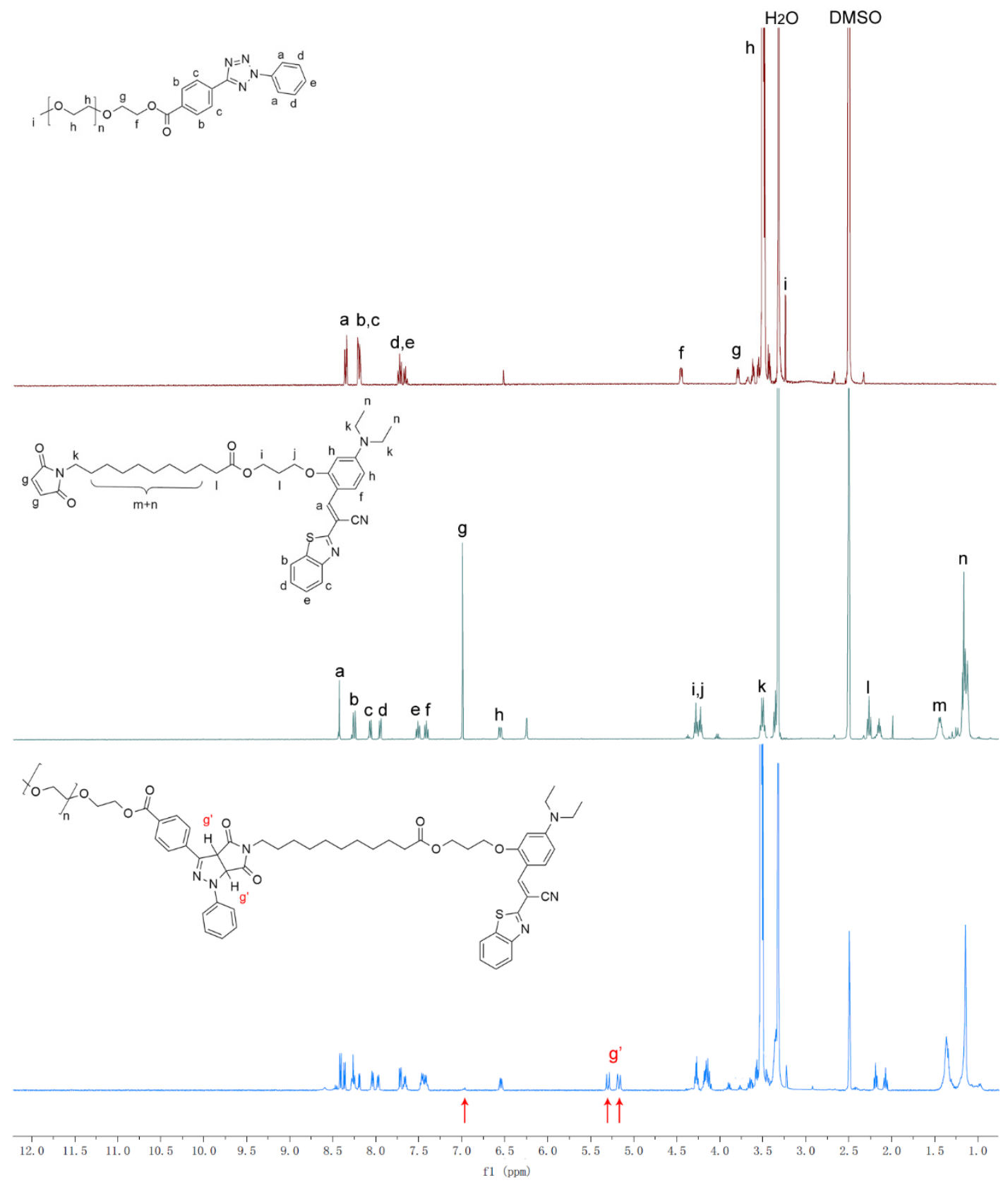

Figure S1. ${ }^{1} \mathrm{H}$ NMR spectral comparison ( $d_{6}$-DMSO) of the two molecular precursors $\left(\mathbf{E}_{45} \mathbf{A z}\right.$ and $\left.\mathbf{M C V}\right)$ and their photoligation adduct $\left(\mathbf{E}_{\mathbf{4 5}} \mathbf{A z}-\mathbf{M C V}\right)$. After photoreaction, the typical alkene proton peak $\left(\mathrm{H}_{\mathrm{g}} \sim 7.02 \mathrm{ppm}\right)$ of maleimide group vanished completely, while a new group of proton $\left(\mathrm{H}_{\mathrm{g}} \sim 5.29\right.$ and $5.21 \mathrm{ppm}$, as indicated by arrows) ascribed to pyrazoline species appeared, ${ }^{7}$ which indicates the full conversion of $\mathbf{E}_{\mathbf{4 5}} \mathbf{A z}$ and $\mathbf{M C V}$ into $\mathbf{E}_{45} \mathbf{A z}-\mathbf{M C V}$ conjugate. 

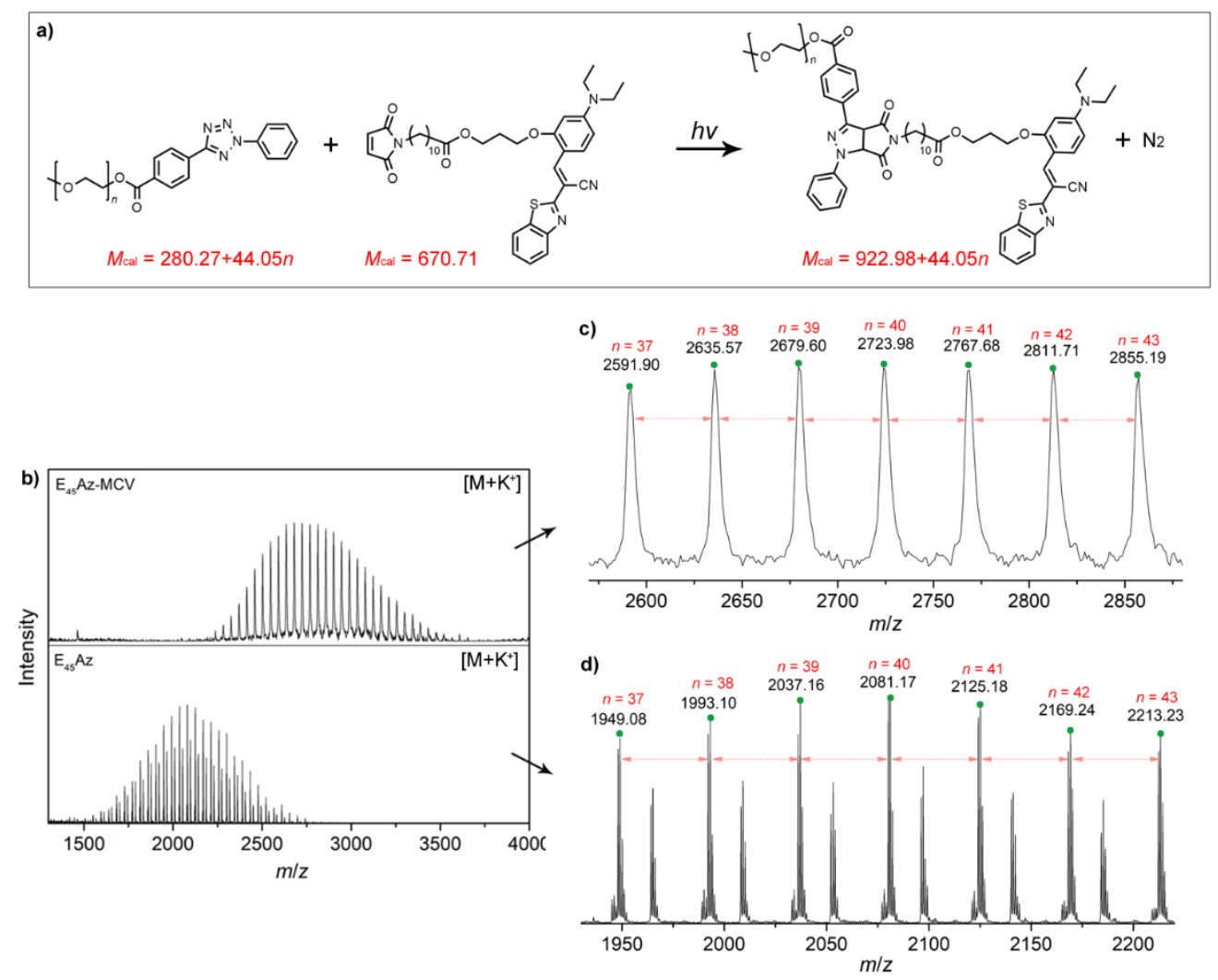

Figure S2. (a) Schematic illustration of the photoclick reaction $\left(\mathbf{E}_{n} \mathbf{A z}+\mathbf{M C V} \rightarrow \mathbf{E}_{n} \mathbf{A z}-\right.$ $\mathbf{M C V}+\mathrm{N}_{2}$ ) and the theoretical molecular weights: $\mathbf{E}_{n} \mathbf{A z}, M_{\mathrm{cal}}=280.27+44.05 n ; \mathrm{MCV}$, $M_{\text {cal }}=670.71 ; \mathbf{E}_{\boldsymbol{n}} \mathbf{A z}-\mathbf{M C V}, M_{\mathrm{cal}}=922.98+44.05 n$. (b) The measured MALDI-TOF mass spectra of the molecular precursor $\left(\mathbf{E}_{\mathbf{4 5}} \mathbf{A z}\right.$, bottom panel) and final photoclick product ( $\mathbf{E}_{45} \mathbf{A z}-\mathbf{M C V}$, top panel). Peak group of (c) $\mathbf{E}_{45} \mathbf{A z}$ and (d) $\mathbf{E}_{\mathbf{4 5}} \mathbf{A z}-\mathbf{M C V}$ : The difference in molecular mass between two neighboring main peaks is constant at 44 , equal to the molecular weight the polyether repeating unit $\left(-\mathrm{CH}_{2} \mathrm{CH}_{2} \mathrm{O}-\right)$. For the case of $\mathbf{E}_{45} \mathbf{A z}$, the calculated $\left[\mathrm{M}+\mathrm{K}^{+}\right]$should be $1949.22(n=37), 1993.27(n=38), 2037.32$ $(n=39), 2081.37(n=40), 2125.42(n=41), 2169.47(n=42), 2213.52(n=43)$; the actual molecular masses are $1949.08(n=37), 1993.10(n=38), 2037.16(n=39)$, $2081.17(n=40), 2125.18(n=41), 2169.24(n=42), 2213.23(n=43)$. For the case of $\mathbf{E}_{45} \mathbf{A z}-\mathbf{M C V}$, the calculated $\left[\mathrm{M}+\mathrm{K}^{+}\right]$should be $2591.92(n=37), 2635.97(n=38)$, $2680.02(n=39), 2724.07(n=40), 2768.12(n=41), 2812.17(n=42), 2856.22(n=43)$; the actual molecular masses are $2591.90(n=37), 2635.57(n=38), 2679.60(n=39)$, $2723.98(n=40), 2767.68(n=41), 2811.71(n=42), 2855.19(n=43)$. The matching of molecular weights indicate the achievement of photoclick reaction. 


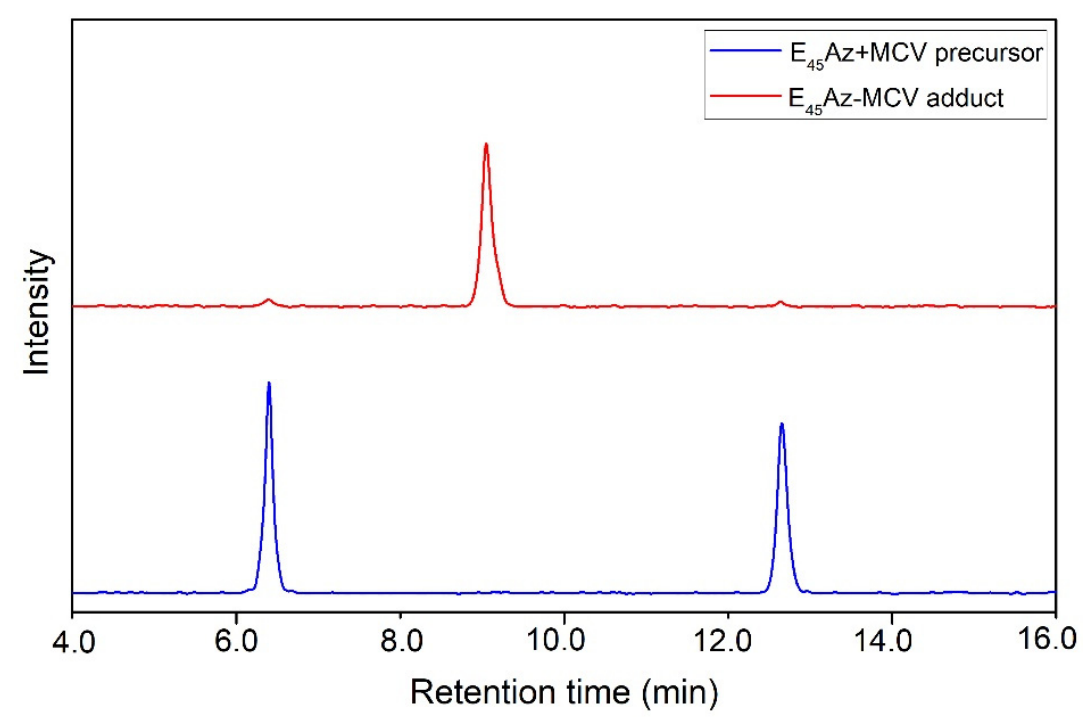

Figure S3. HPLC traces of the two precursors $\left(\mathbf{E}_{45} \mathbf{A z}\right.$ and $\mathbf{M C V}$, blue curve) before photoreaction and the resulting adduct $\left(\mathbf{E}_{\mathbf{4 5}} \mathbf{A z}-\mathbf{M C V}\right.$, red curve) after photoreaction. In the absence of light stimulus, the two strong peaks at the retention time of 6.4 min and 12.7 min were ascribed to the precursors, $\mathbf{E}_{45} \mathbf{A z}$ and $\mathbf{M C V}$, respectively. In the presence of light stimulus for one hour, these two peaks were strongly depressed $(<5 \%)$ whereas a new peak at the retention time of 8.9 min ascribed to the $\mathbf{E}_{\mathbf{4 5}} \mathbf{A z}-\mathbf{M C V}$ adduct was enhanced. This indicate the photoclick reaction can reach nearly full conversion $(>95 \%)$. 


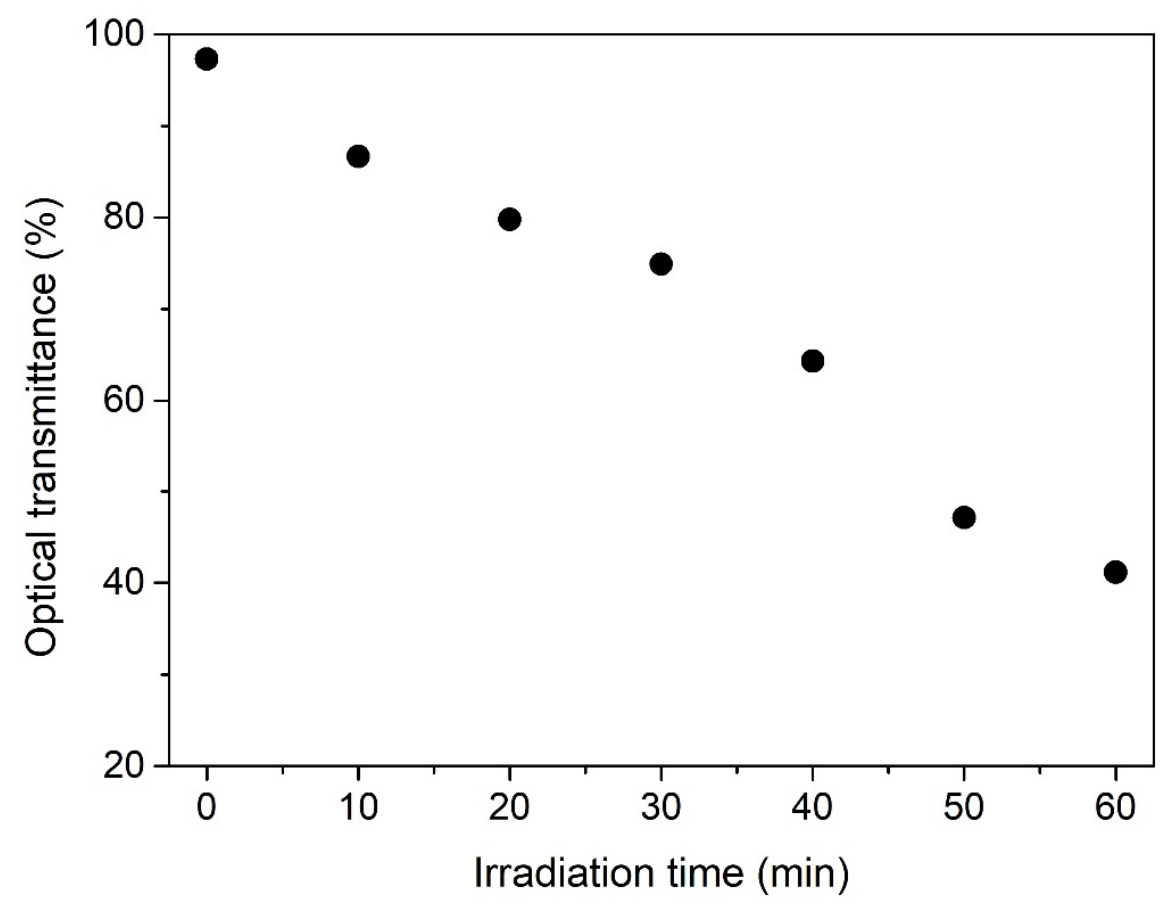

Figure S4. Optical transmittance variation against the photoreaction time monitored by $\mathrm{UV}$-vis spectroscopy using a wavelength of $600 \mathrm{~nm}$. Before light irradiation, the $\mathbf{E}_{45} \mathbf{A z}$ and MCV mixture solution $(1 / 1,35 \mathrm{mM})$ showed $97 \%$ optical transmittance, whereas its solution transmittance decreased to $41 \%$ after light irradiation. Such an increase in solution turbidity suggested the direct formation of large aggregates in solution. 


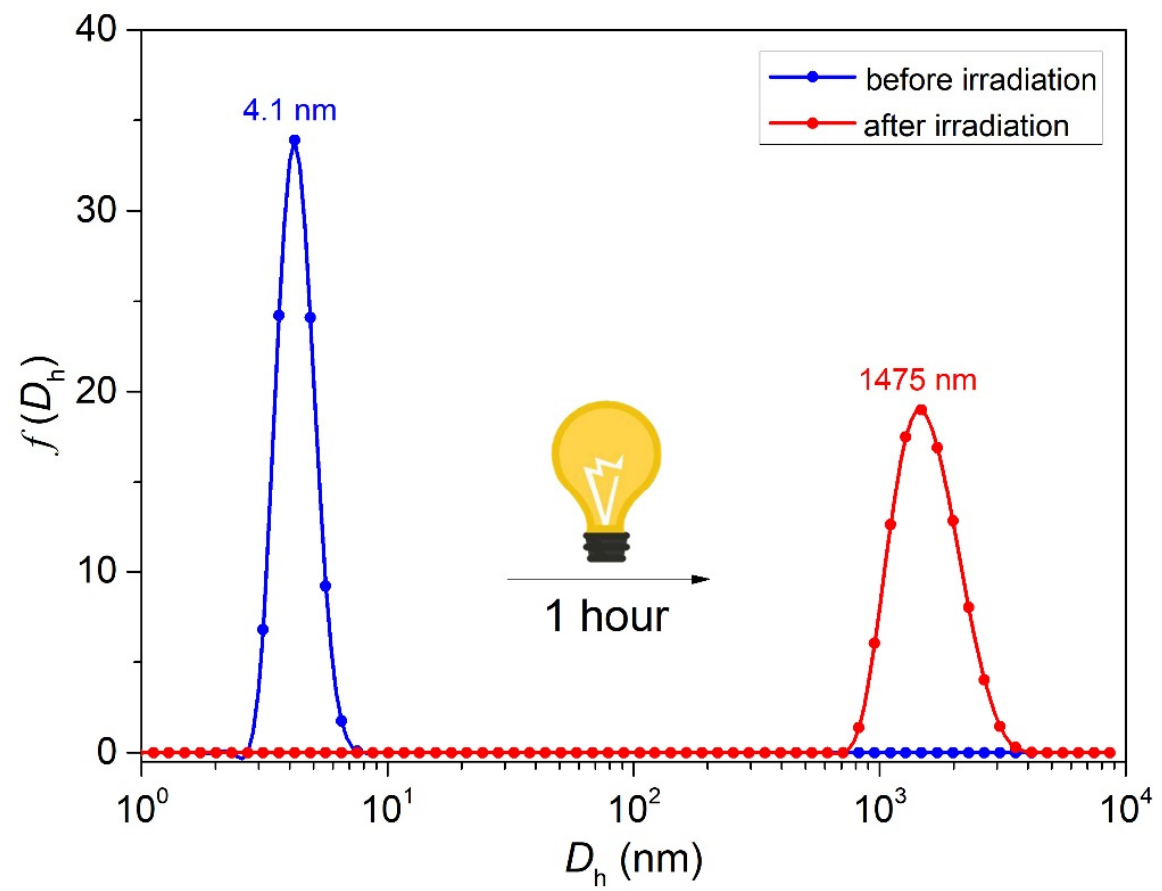

Figure S5. DLS results showing the change of hydrodynamic diameter $\left(D_{\mathrm{h}}\right)$ before and after light irradiation for one hour. The initial size of the $\mathbf{E}_{\mathbf{4 5}} \mathbf{A z}$ and $\mathbf{M C V}$ in solution without photoreaction is determined to be $4.1 \mathrm{~nm}$, which is consistent with the actual molecular length of $\mathbf{E}_{\mathbf{4 5}} \mathbf{A z}$. After photoreaction, the theoretical molecular length of the $\mathbf{E}_{45} \mathbf{A z}-\mathbf{M C V}$ adduct should be smaller than $5 \mathrm{~nm}$; however, the measured value is 1475 $\mathrm{nm}$, which further indicates that the resulting $\mathbf{E}_{\mathbf{4 5}} \mathbf{A z}-\mathbf{M C V}$ adduct in-situ self-assemble into large aggregates. 


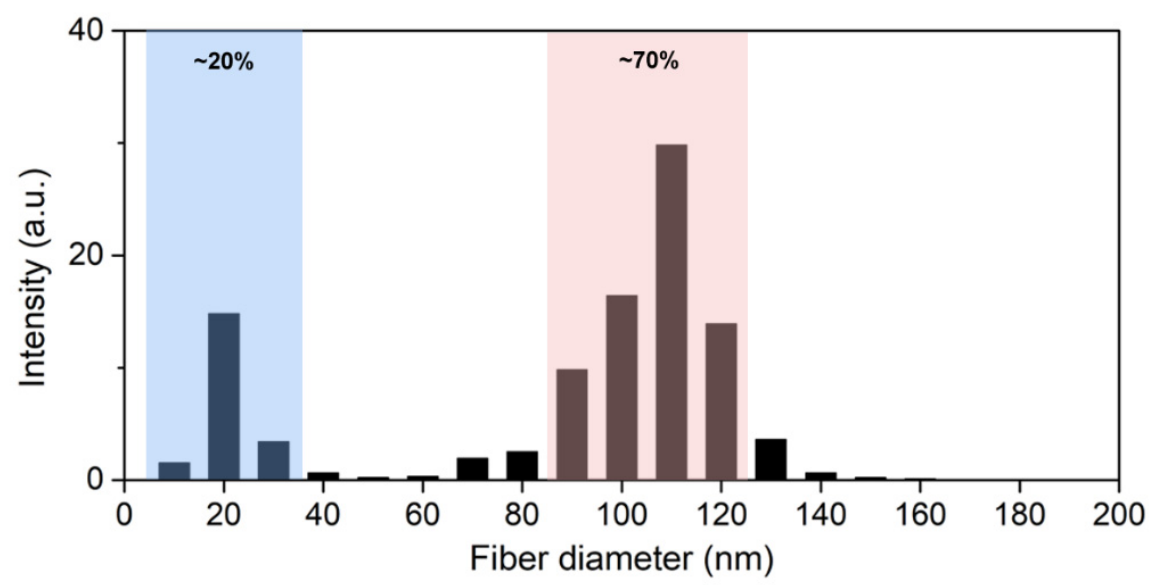

Figure S6. Fiber diameter distribution obtained from TEM particle statistics software by using 280 discrete nanofibers. It can be found that there exist two different classes of nanofibers, one of which is thin fiber with $\sim 20 \mathrm{~nm}$ of diameter that accounts for $\sim 20 \%$ of total population (blue region), the other of which is thick fiber with $90 \sim 120 \mathrm{~nm}$ of diameter that accounts for $\sim 70 \%$ of total population (red region). 
Confocal Laser Scanning Microscopy (CLSM) for the Fluorescent Superhelical Fiber Structure.

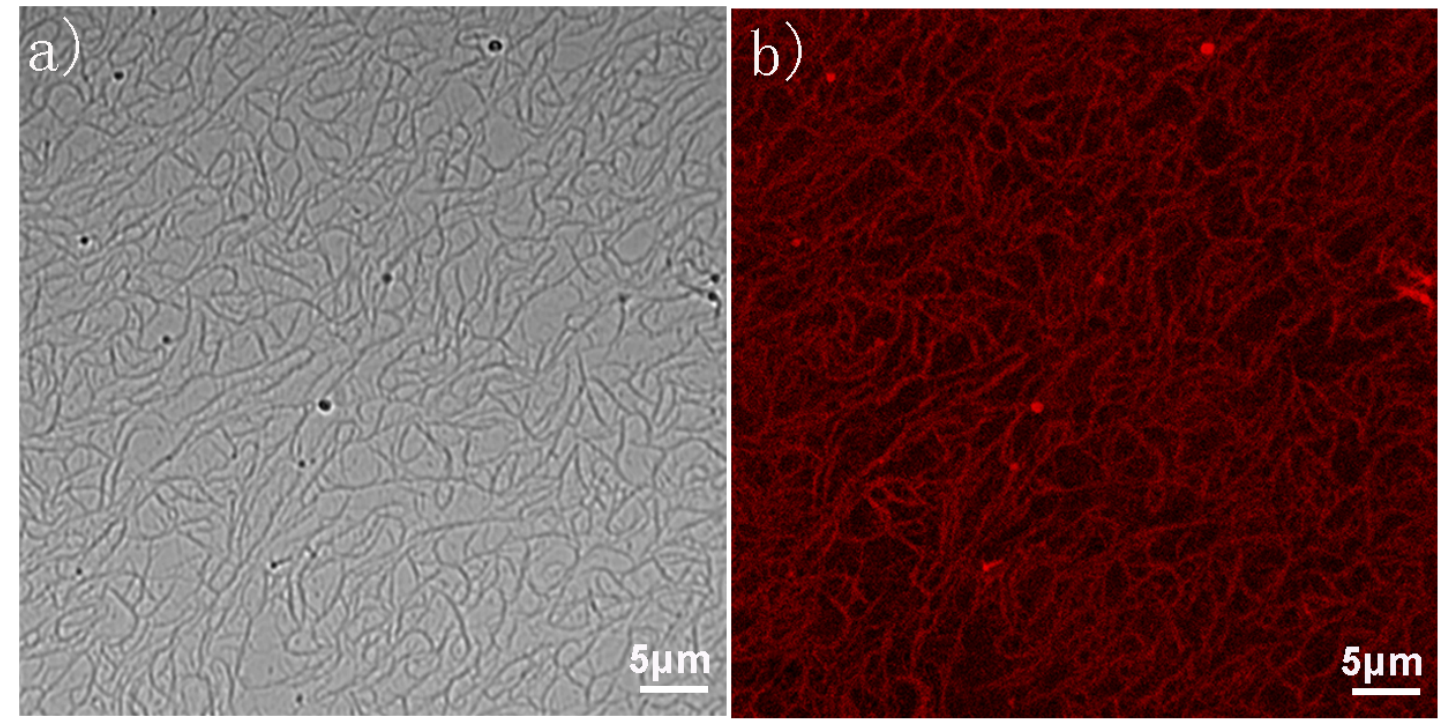

Figure S7. CLSM image showing the superhelical nanofibers: (a) bright field and (b) red channel. 
Photoclick Reaction Kinetics.

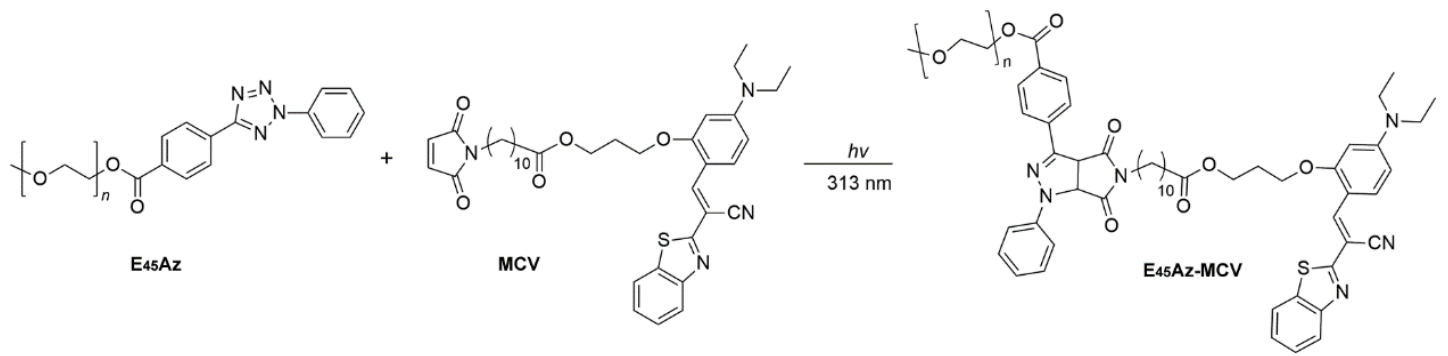

Using $\mathbf{E}_{\mathbf{4 5}} \mathbf{A z}$ as the model compound, $12.0 \mathrm{mg}$ of $\mathbf{E}_{\mathbf{4 5}} \mathbf{A z}$ ( $0.0053 \mathrm{mmol}, 1.0$ equiv.) and $4 \mathrm{mg}$ of MCV (0.0064 mol, 1.2 equiv.) were dissolved in $\mathrm{THF} / \mathrm{H}_{2} \mathrm{O}$ solution $\left(\mathrm{THF} / \mathrm{H}_{2} \mathrm{O}\right.$, $\mathrm{v} / \mathrm{v}, 4 / 1$, total volume of $8 \mathrm{~mL}$ ). The solution were deoxygenated by purging the vial with nitrogen for $10 \mathrm{~min}$. The sample was irradiated with stirring for $75 \mathrm{~min}$ in the aforementioned photoreaction equipment and the aliquots of samples were taken out at a constant interval of 5 min to analyze the conversion.
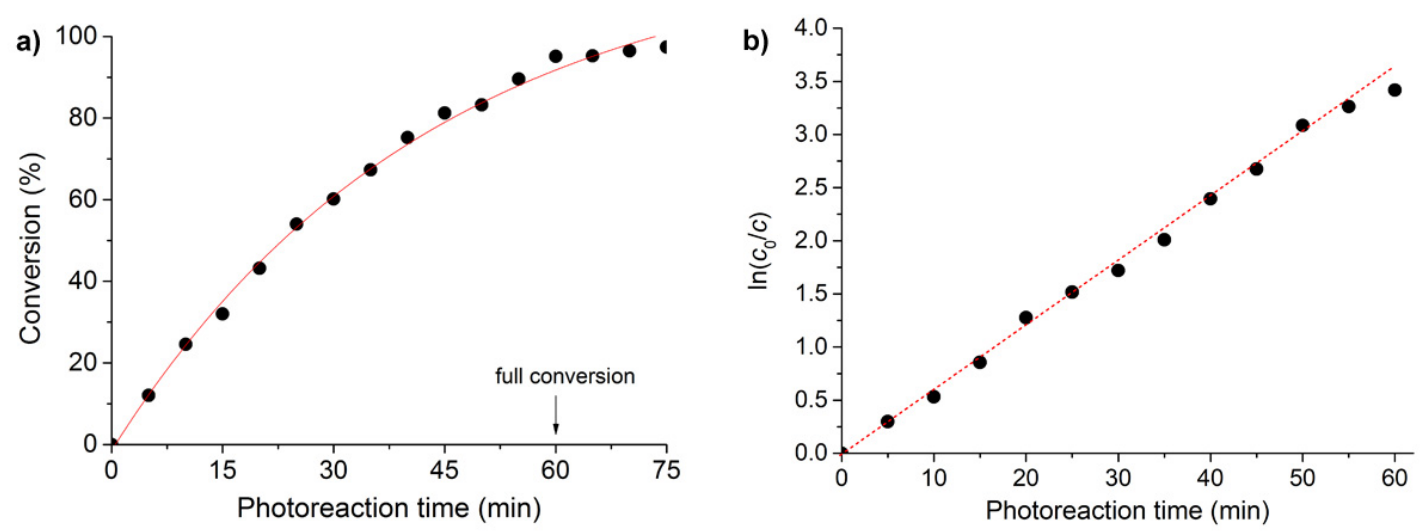

Figure S8. (a) Conversion vs. photoreaction time plot of the molecular precursor $\mathbf{E}_{45} \mathbf{A z}$ when reacted with $\mathrm{MCV}$ in $\mathrm{THF} / \mathrm{H}_{2} \mathrm{O}$ solution. (b) Pseudo-first order reaction curve for the reaction between $\mathbf{E}_{\mathbf{4 5}} \mathbf{A z}$ and $\mathbf{M C V}$ in $\mathrm{THF} / \mathrm{H}_{2} \mathrm{O}$ solution. The kinetic data follows a pseudo-first order kinetics owing to the linear fitting mode and based on these results the rate coefficient for this photoclick reaction is evaluated to be $k_{\mathrm{c}}=4.52 \times 10^{-4} \mathrm{~s}^{-1}$. Such a pseudo-first order reaction kinetics is helpful to control the photoreaction extent, in order to regulate the self-assembly and manipulate the morphological evolution. 
TEM Showing the Initial Morphology.

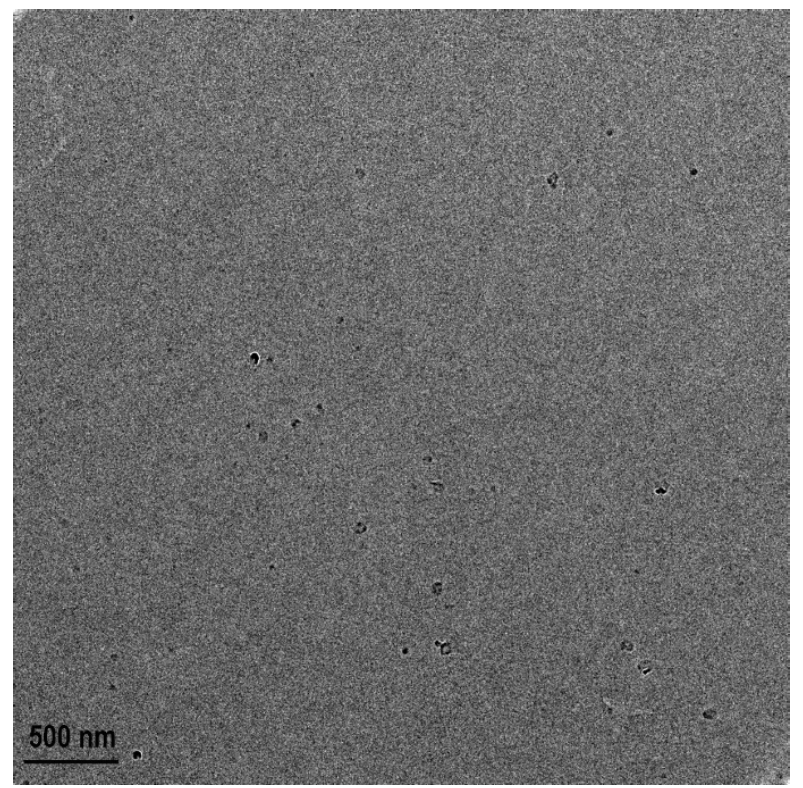

Figure S9. TEM image of the reaction mixture at the beginning of UV irradiation ( 0 $\min$ ) and no regular aggregates were observed. 


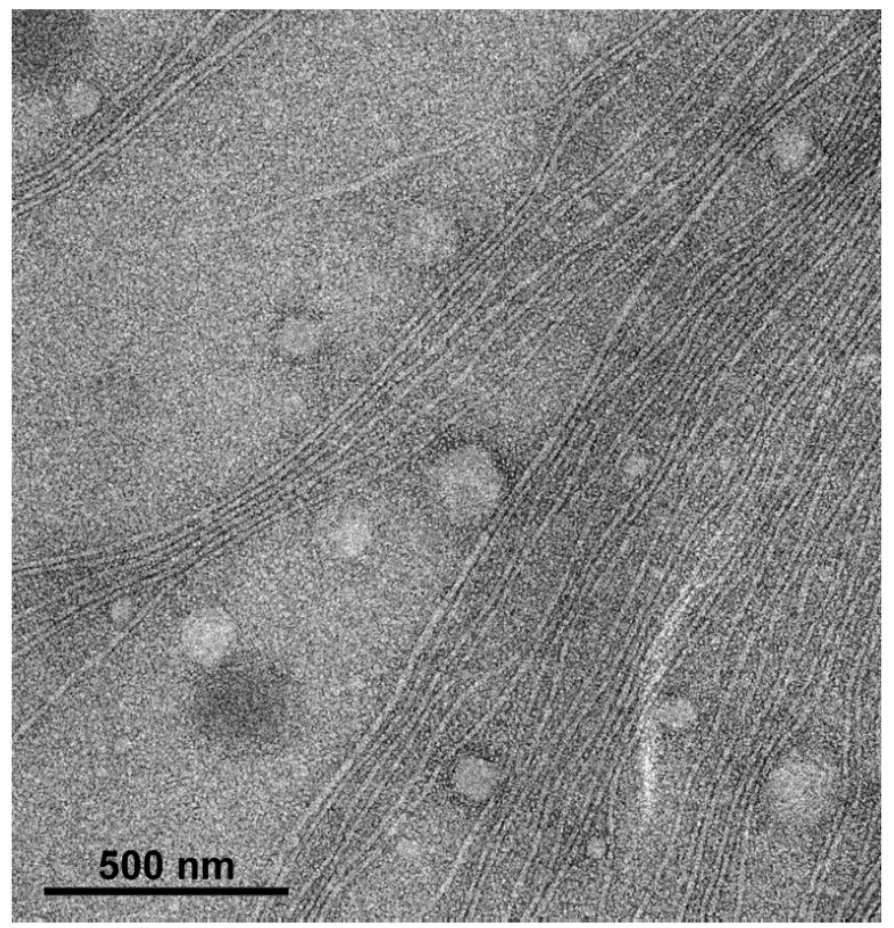

Figure S10. The $\mathbf{E}_{\mathbf{4 5}} \mathbf{A z}-\mathbf{M C V}$ filamentous structure. Their smooth filament surface in axial direction indicate that they are non-helical shape and their length is over several micrometers. 

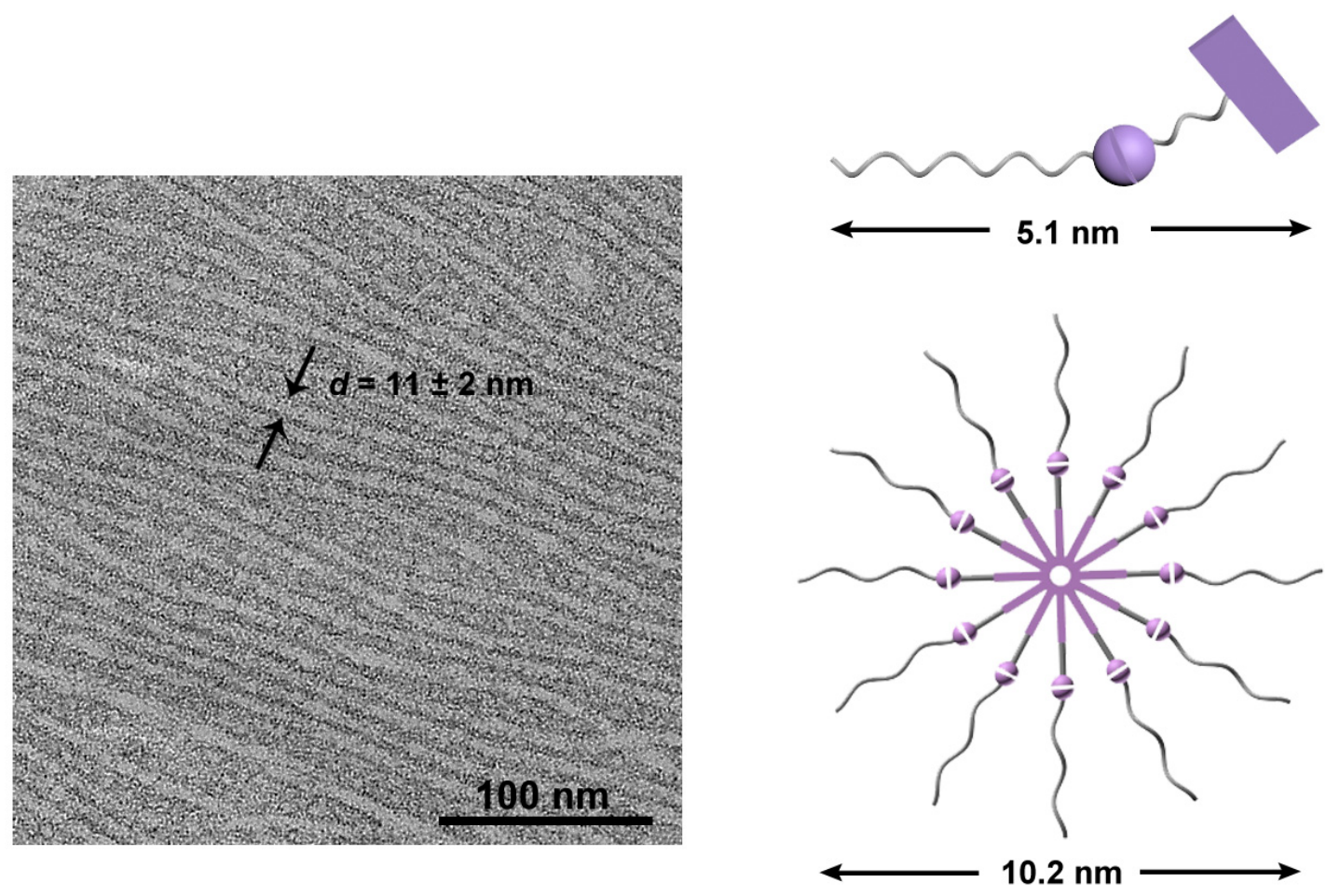

Figure S11. Magnified TEM image showing the filamentous width is $(11 \pm 2) \mathrm{nm}$ (left panel). This result is close to the double molecular length of $\mathbf{E}_{\mathbf{4 5}} \mathbf{A z}-\mathbf{M C V}(5.1 \times 2=$ $10.2 \mathrm{~nm}$ ), which illustrates that the nanofilament may adopt the bimolecularly columnar arrangement (right panel). 


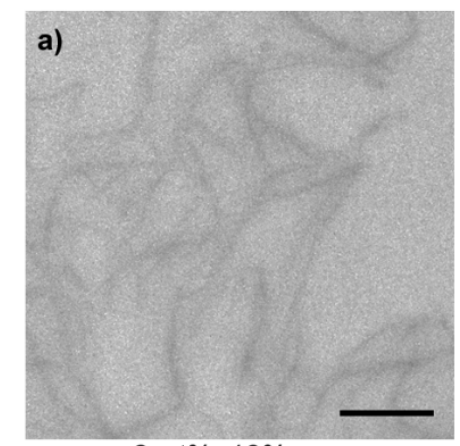

2 wt $\%, 18 \%$ conv.

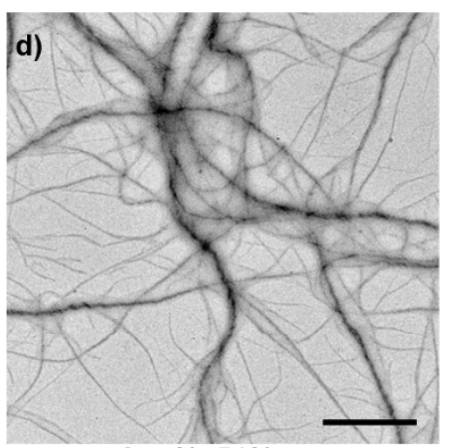

2 wt $\%, 51 \%$ conv.

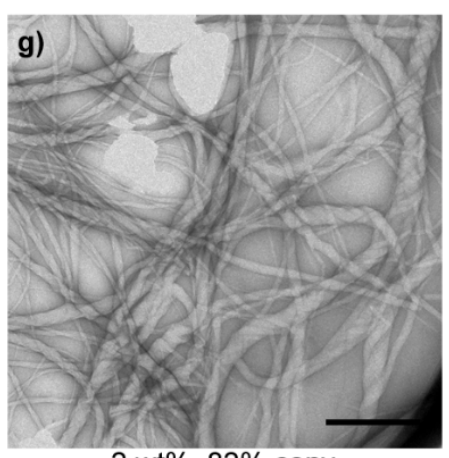

2 wt $\%, 82 \%$ conv.

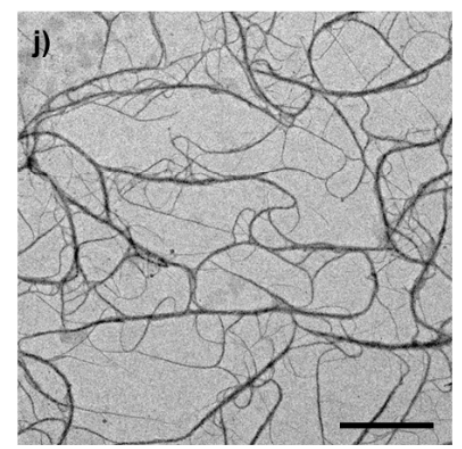

8 wt $\%, 36 \%$ conv.

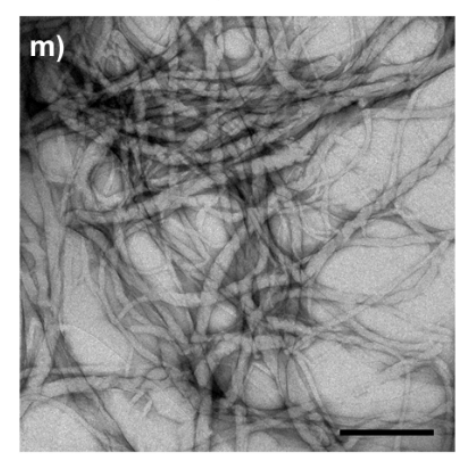

8 wt $\%, 78 \%$ conv.

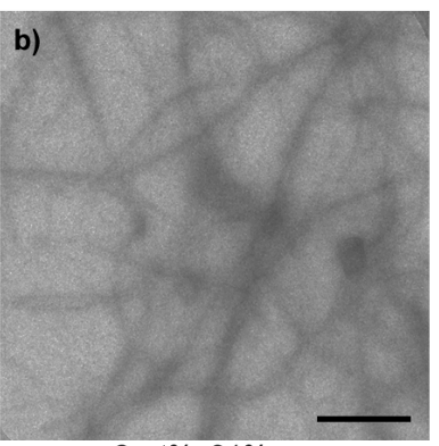

2 wt $\%, 31 \%$ conv.

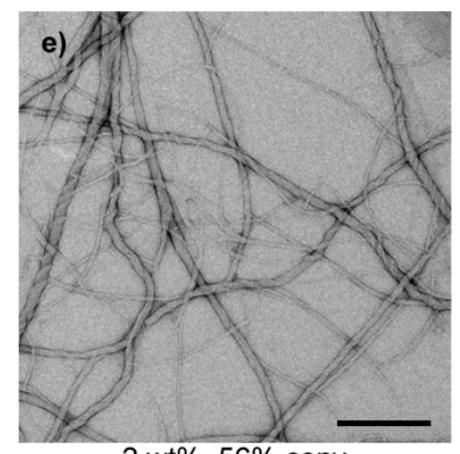

2 wt $\%, 56 \%$ conv.

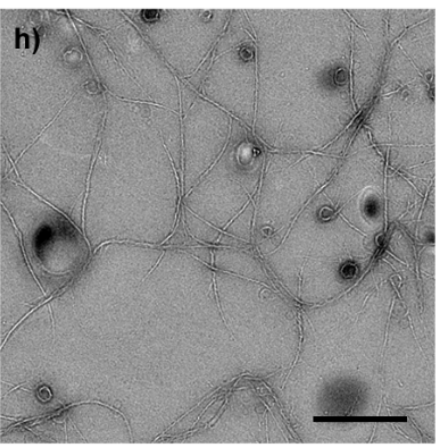

8 wt $\%, 12 \%$ conv.

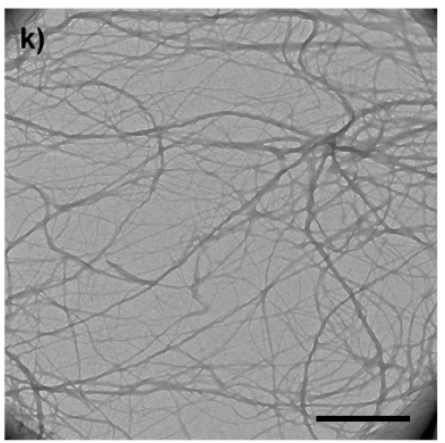

$8 \mathrm{wt} \%, 46 \%$ conv.

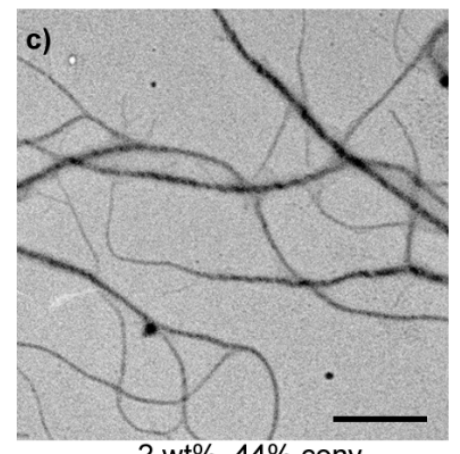

2 wt $\%, 44 \%$ conv.
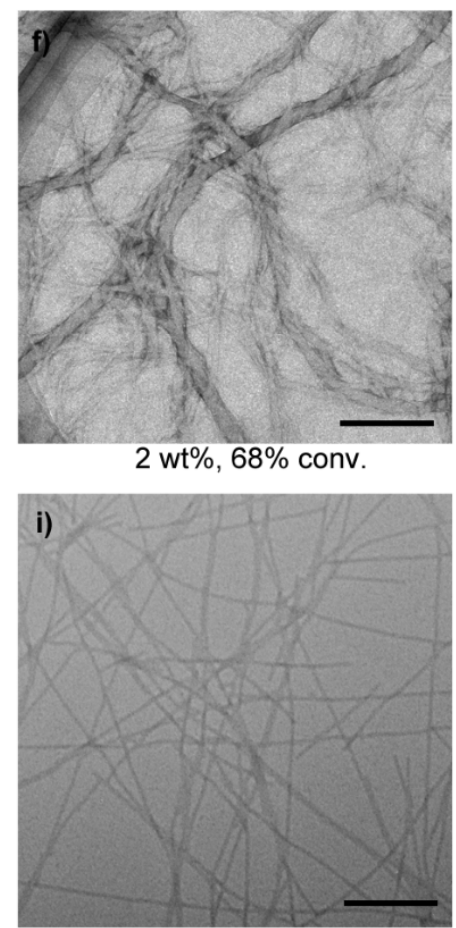

$8 w t \%, 24 \%$ conv

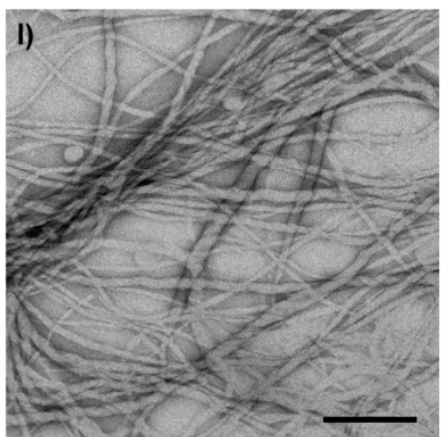

8 wt $\%, 65 \%$ conv. 
Figure S12. Representative morphologies of light-click in-situ self-assembly (LISA) of $\mathbf{E}_{45} \mathbf{A z}-\mathbf{M C V}$ in various solid concentrations ( $2 \mathrm{wt} \%$ and $8 \mathrm{wt} \%$ ) and photoreaction conversion: (a) $2 \mathrm{wt} \%, 18 \%$ conv., worm-like micelles; (b) $2 \mathrm{wt} \%, 31 \%$ conv., filaments; (c) $2 \mathrm{wt} \%, 44 \%$ conv., single-stranded helical fibrils; (d) $2 \mathrm{wt} \%, 51 \%$ conv., singlestranded helical fibrils; (e) $2 \mathrm{wt} \%, 56 \%$ conv., single-stranded helical fibrils and multistranded helical fibrils; (f) $2 \mathrm{wt} \%, 68 \%$ conv., multi-stranded helical fibrils, (g) $2 \mathrm{wt} \%$, $82 \%$ conv., superhelical fibers; (h) $8 \mathrm{wt} \%, 12 \%$ conv., worm-like micelles; (i) $8 \mathrm{wt} \%$, 24\% conv., filaments; (j) 8 wt\%, 36\% conv., single-stranded helical fibrils; (k) 8 wt\%, $46 \%$ conv., single-stranded helical fibrils; (1) $8 \mathrm{wt} \%$, 65\% conv., multi-stranded helical fibrils; (m) 8 wt\%, 78\% conv., multi-stranded helical fibrils and superhelical fibers. Scale bar is $200 \mathrm{~nm}$. 


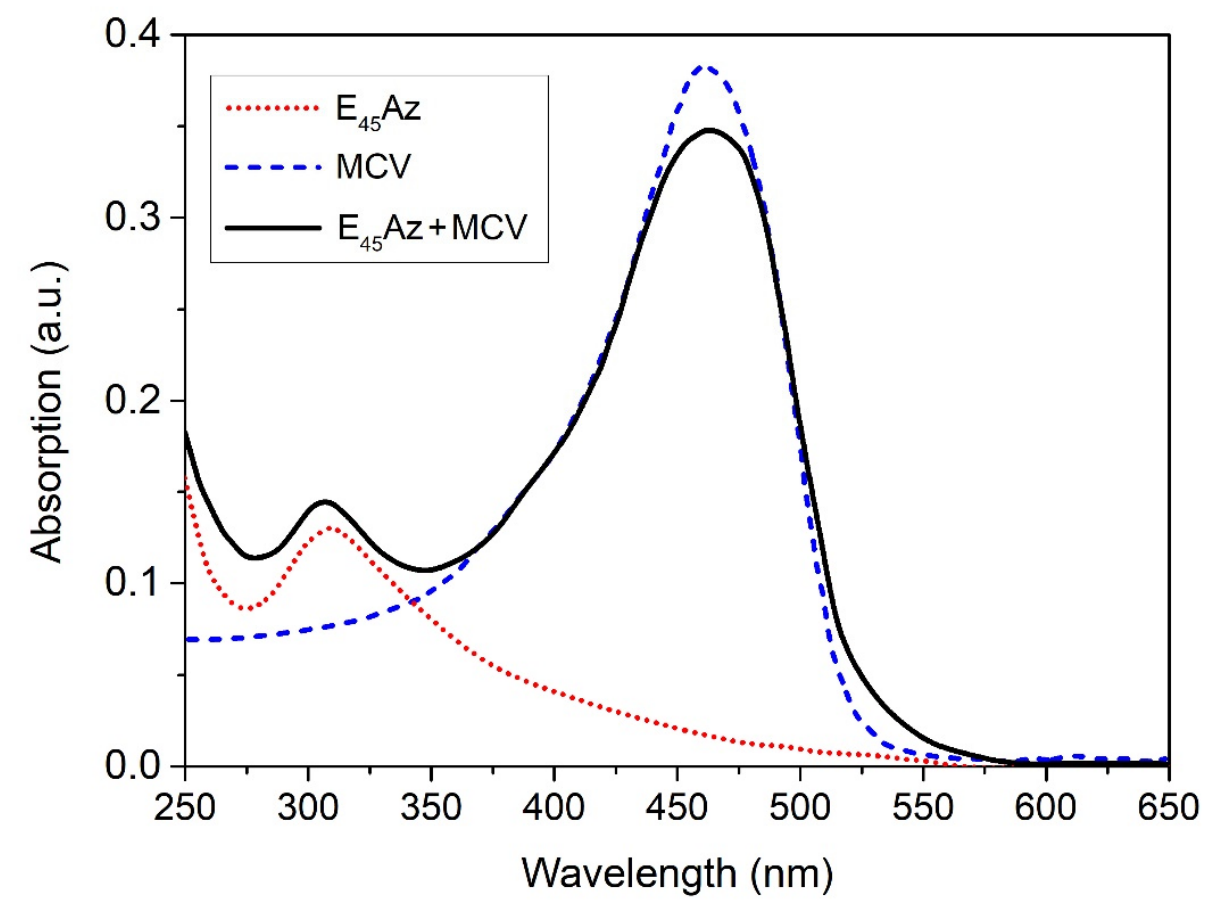

Figure S13. UV-vis spectra of $\mathbf{E}_{\mathbf{4 5}} \mathbf{A z}$ (red dot line), $\mathbf{M C V}$ (blue dash line), and $\mathbf{E}_{\mathbf{4 5}} \mathbf{A z}$ and MCV solution (1/1, black solid line). $\mathbf{E}_{\mathbf{4 5}} \mathbf{A z}$ showed a weak absorption band around $300 \mathrm{~nm}$ ascribed to the tetrazole species, while MCV showed a characteristic absorption at $464 \mathrm{~nm}$ ascribed to the cyanovinylene species. Their binary mixture $\left(\mathbf{E}_{\mathbf{4 5}} \mathbf{A z}+\mathbf{M C V}\right)$ with equal ratio in the absence of light irradiation mainly showed the MCV absorption at $464 \mathrm{~nm}$. 


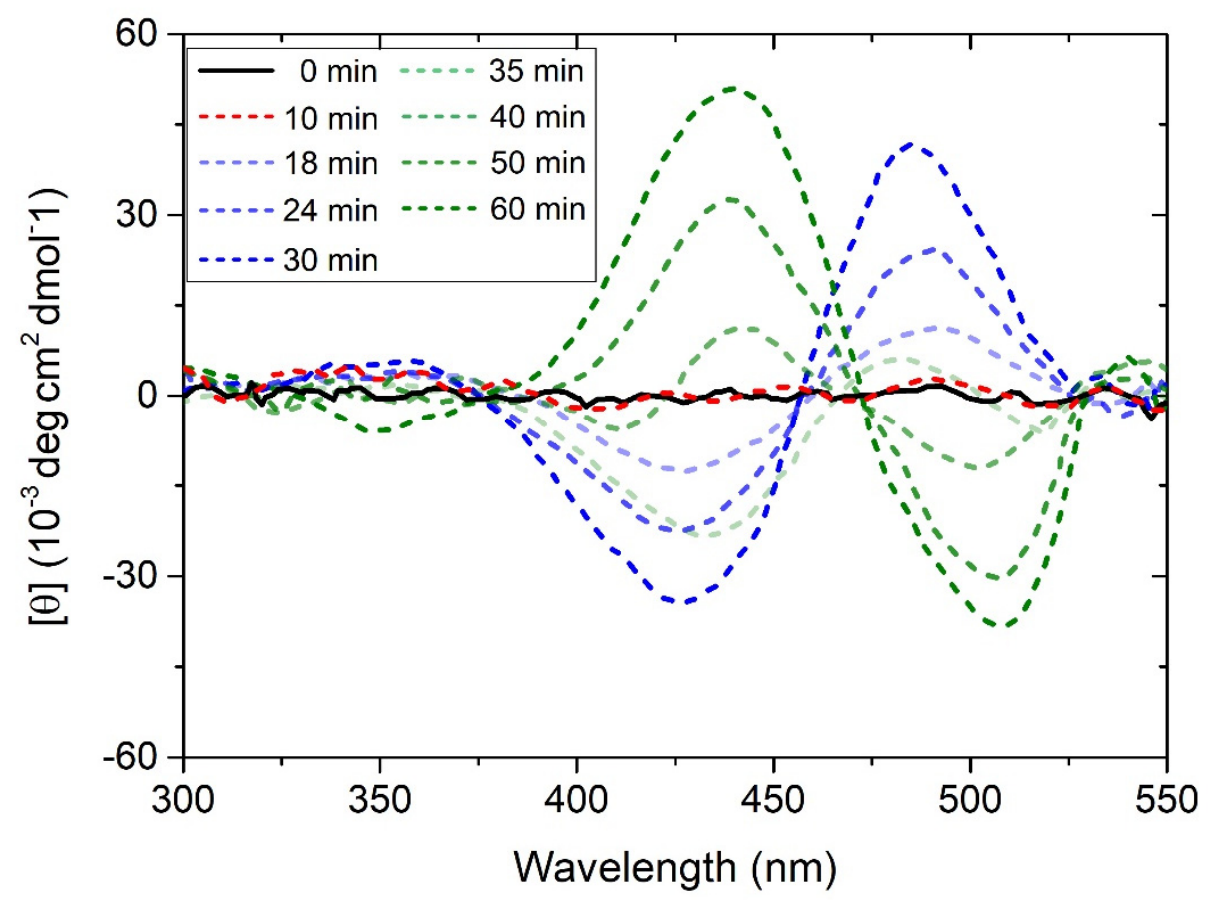

Figure S14. CD spectral variation of $\mathbf{E}_{\mathbf{4 5}} \mathbf{A z}$ and $\mathbf{M C V}$ with light irradiation time: 0 min (black solid line), $10 \mathrm{~min}$ (red dash line), 18, 24 and $30 \mathrm{~min}$ (blue dash lines in different shades), 35, 40, 50 and 60 min (green dash lines in different shades). 


\section{Structural Characterization of All Synthetic Samples.}

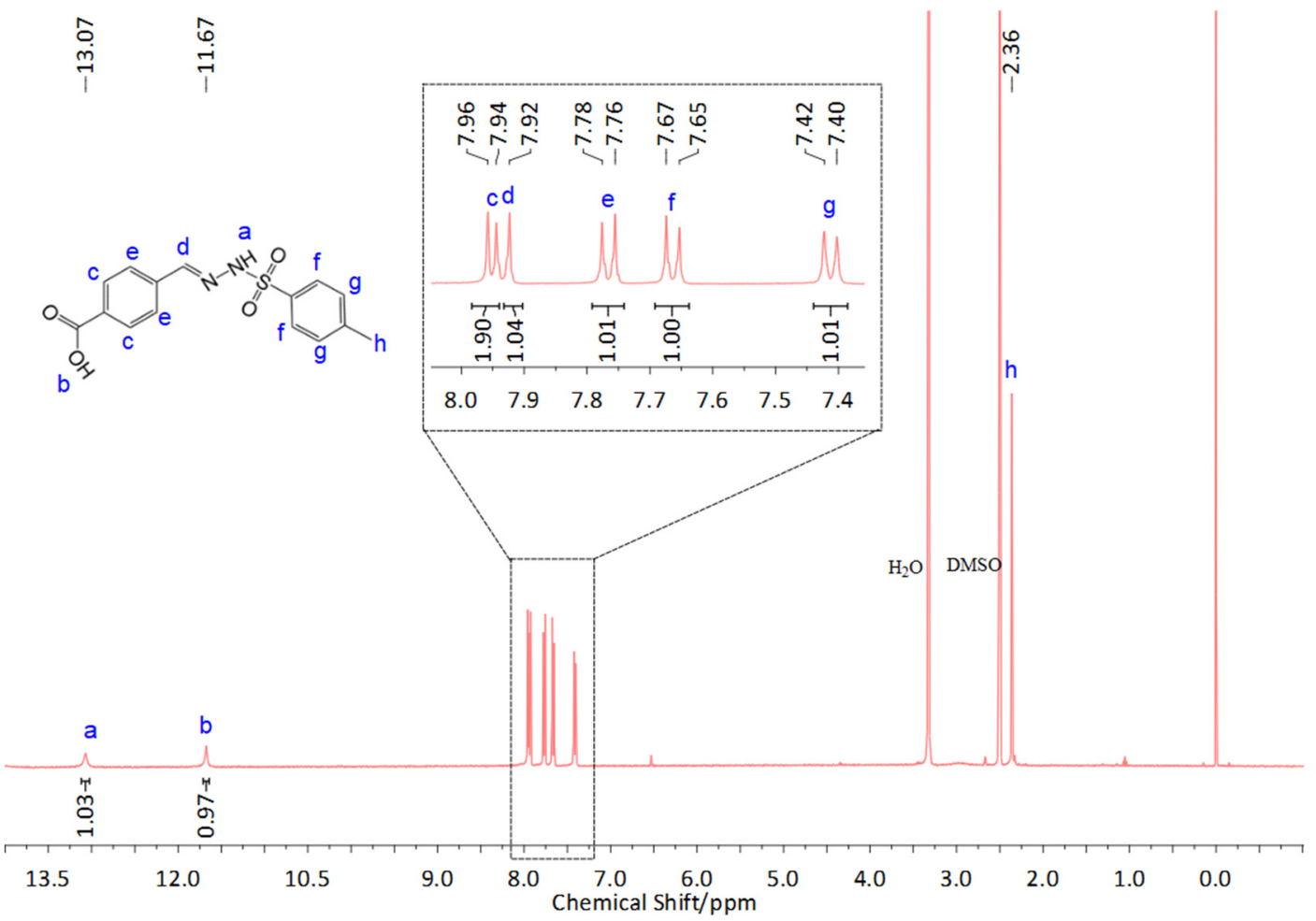

Figure S15. ${ }^{1} \mathrm{H}$ NMR spectrum of (E)-4-((2-tosylhydrazineylidene)methyl)benzoic acid in $d_{6}$-DMSO.

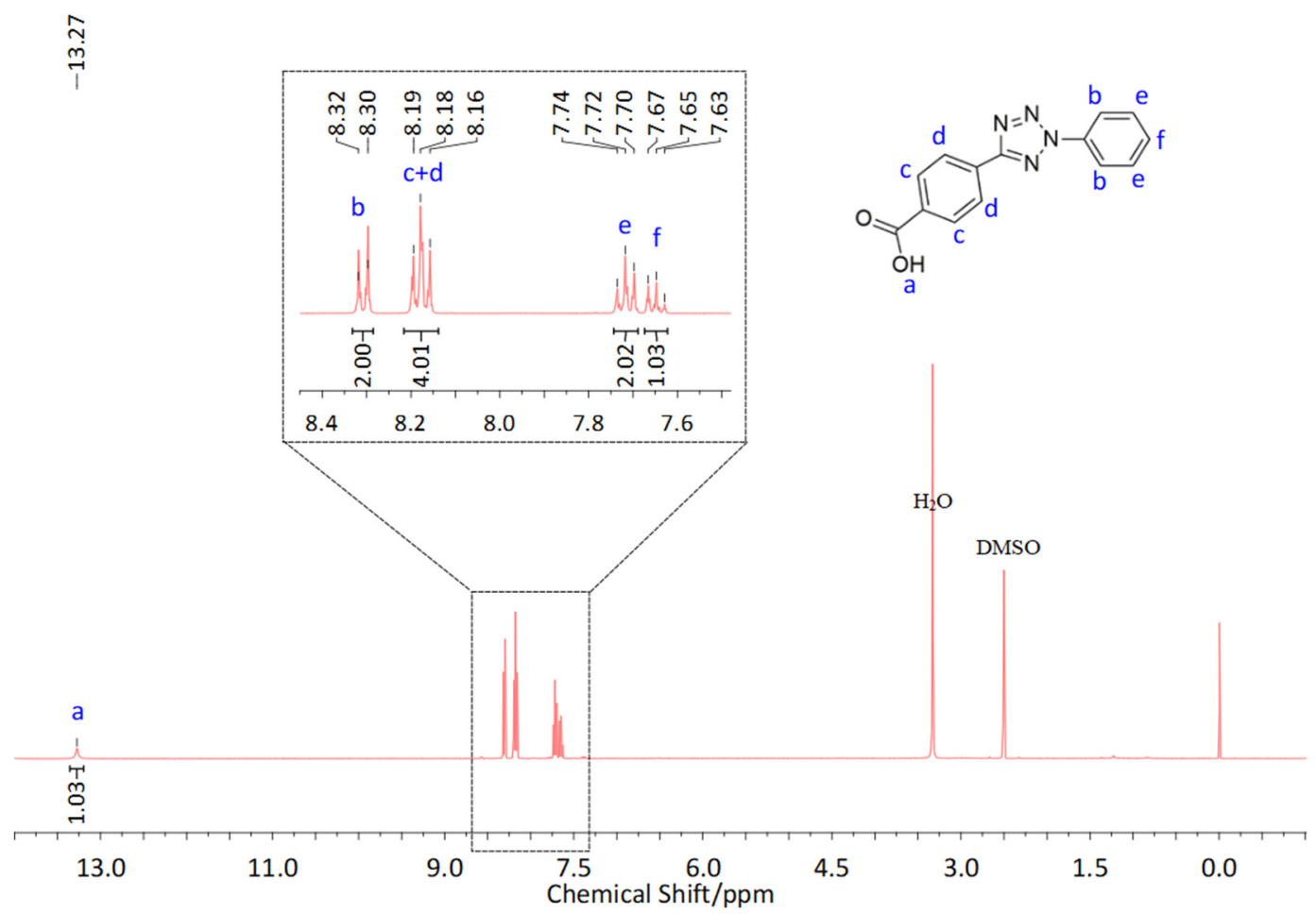

Figure S16. ${ }^{1} \mathrm{H}$ NMR spectrum of tetrazole-derived benzoic acid (Az) in $d 6-\mathrm{DMSO}$. 


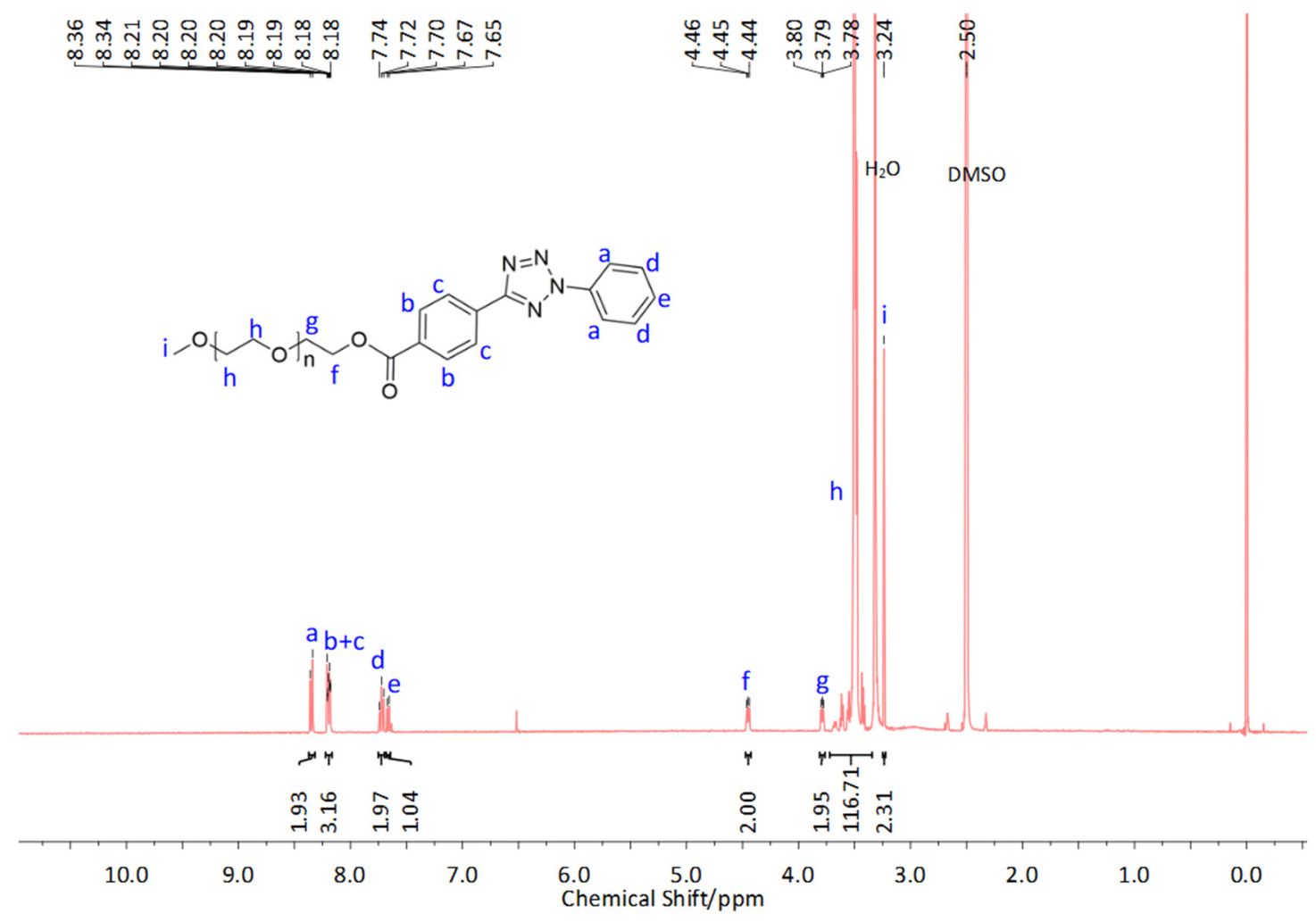

Figure S17. ${ }^{1} \mathrm{H}$ NMR spectrum of the molecular precursor $\mathbf{E}_{\mathbf{4 5}} \mathbf{A z}$ in $d 6$-DMSO.

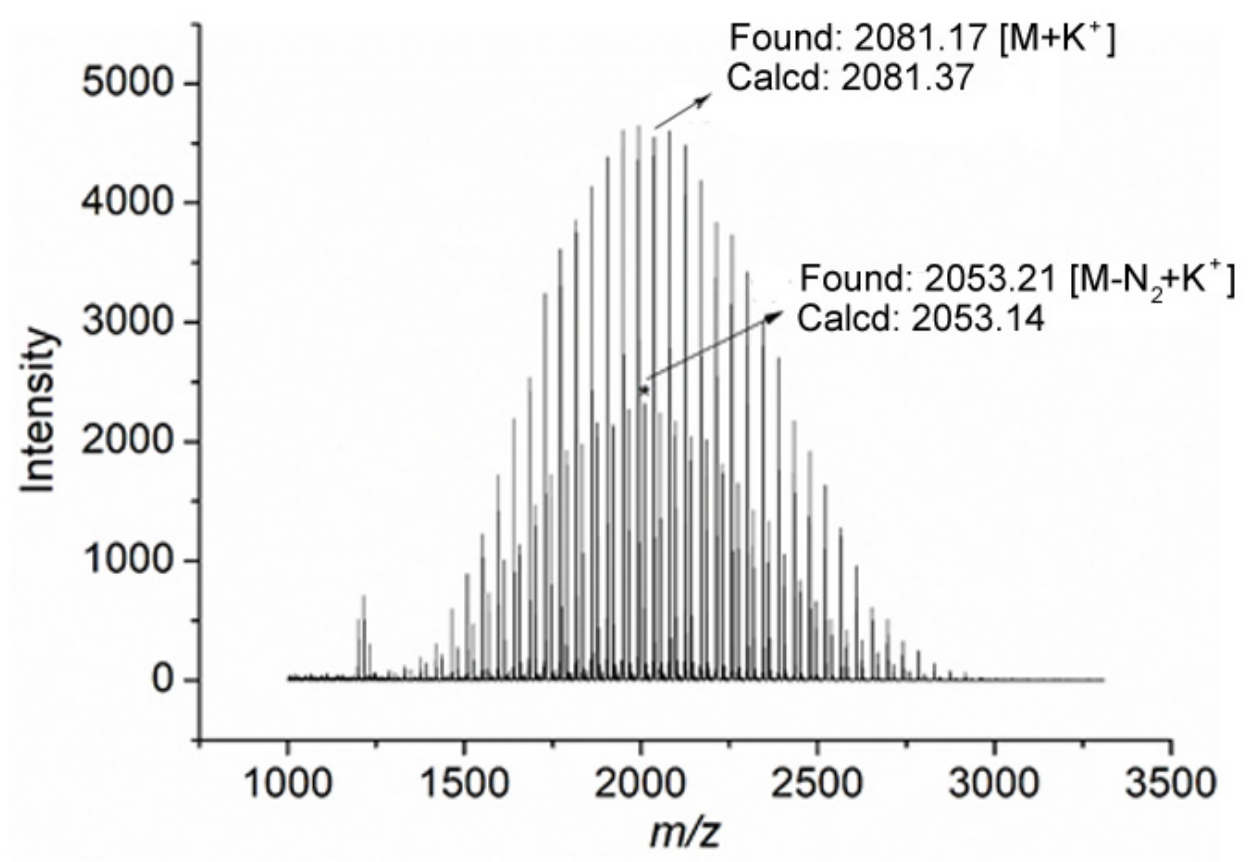

Figure S18. MALDI-TOF mass spectrum of $\mathbf{E}_{\mathbf{4 5}} \mathbf{A z}$. 


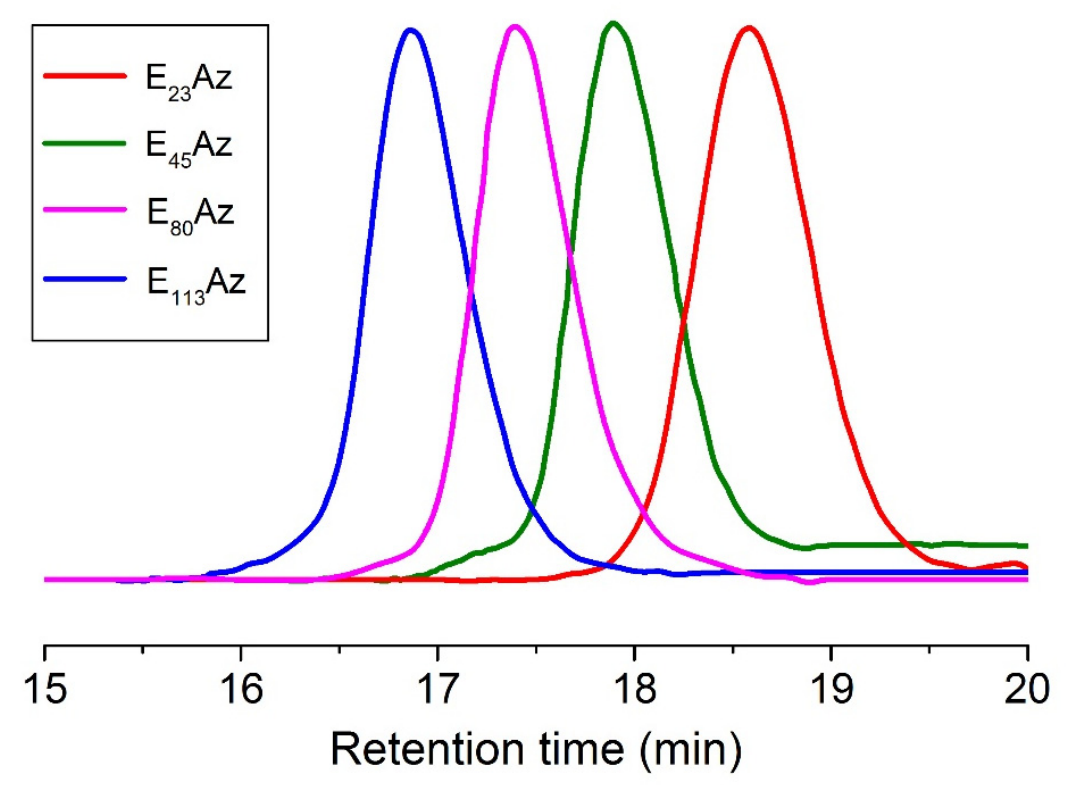

Figure S19. GPC traces for $\mathbf{E}_{113} \mathbf{A z}\left(M_{\mathrm{n}, \mathrm{GPC}}=5170 \mathrm{~g} / \mathrm{mol}, \emptyset=1.03\right), \mathbf{E}_{\mathbf{8 0}} \mathbf{A z}\left(M_{\mathrm{n}, \mathrm{GPC}}=\right.$ $3740 \mathrm{~g} / \mathrm{mol}, \emptyset=1.02), \mathbf{E}_{\mathbf{4 5}} \mathbf{A z}\left(M_{\mathrm{n}, \mathrm{GPC}}=2240 \mathrm{~g} / \mathrm{mol}, \emptyset=1.02\right)$, and $\mathbf{E}_{\mathbf{2 3}} \mathbf{A z}\left(M_{\mathrm{n}}=1590\right.$ $\mathrm{g} / \mathrm{mol}, \emptyset=1.01)$.
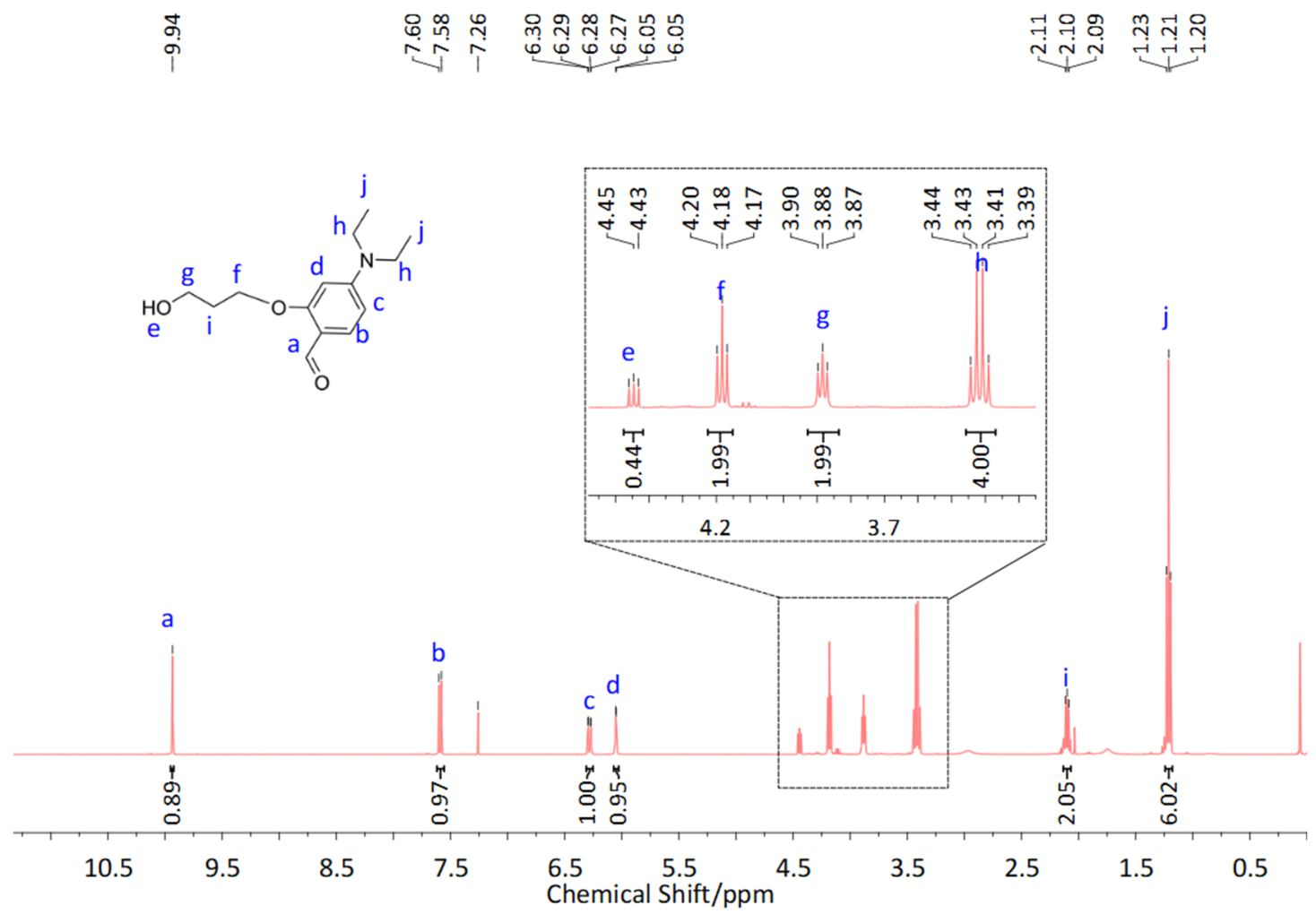

Figure S20. ${ }^{1}$ H NMR spectrum of 4-(diethylamino)-2-(3-hydroxypropoxy) benzaldehyde in $\mathrm{CDCl}_{3}$. 


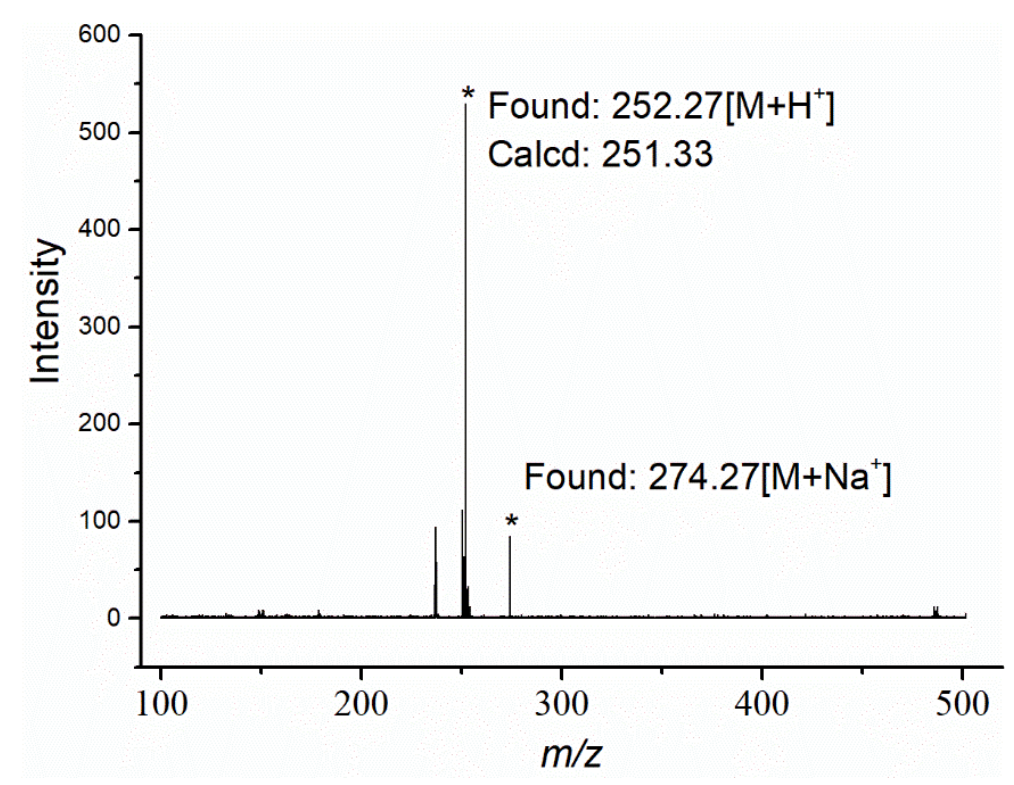

Figure S21. MALDI-TOF mass spectrum of 4-(diethylamino)-2-(3-hydroxypropoxy) benzaldehyde.
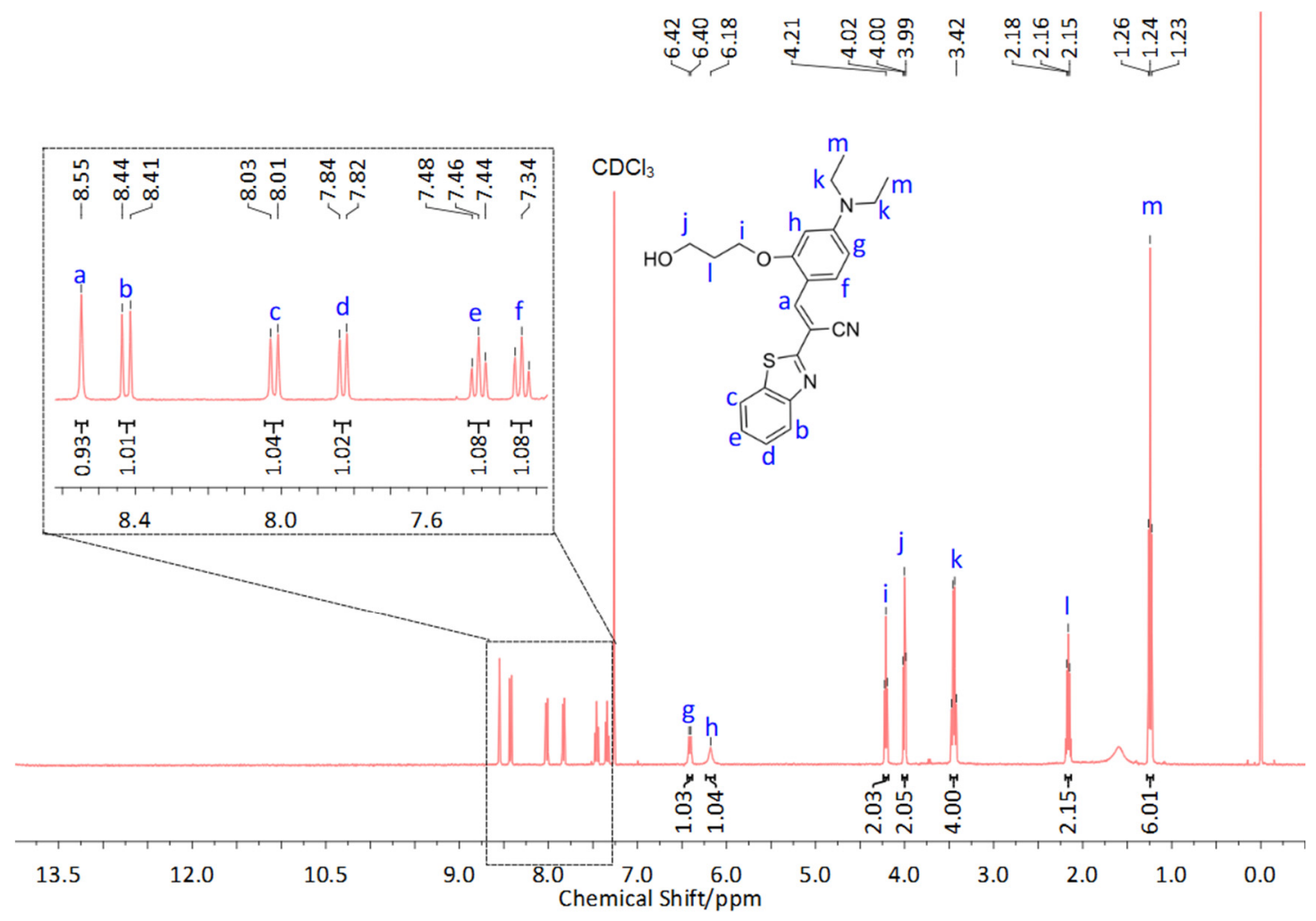

Figure S22. ${ }^{1} \mathrm{H}$ NMR spectrum of cyanovinylene fluorophore $(\mathrm{CV})$ in $\mathrm{CDCl}_{3}$. 


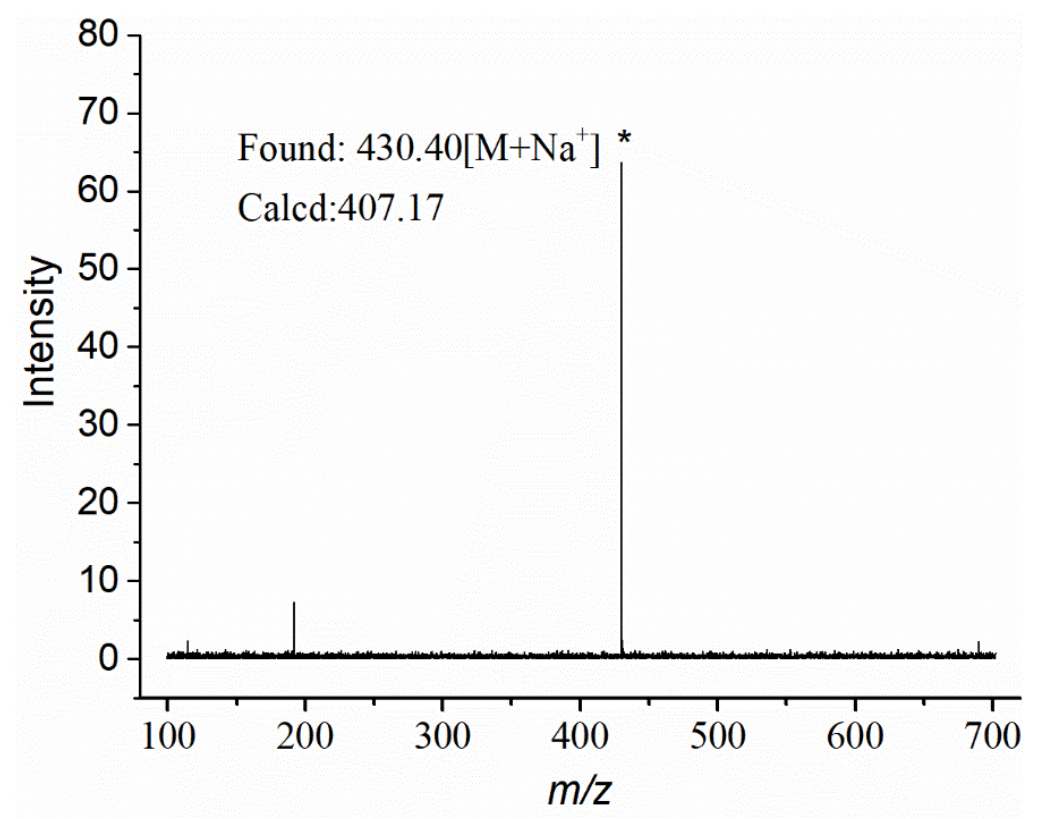

Figure S23. MALDI-TOF mass spectrum of cyanovinylene fluorophore (CV).

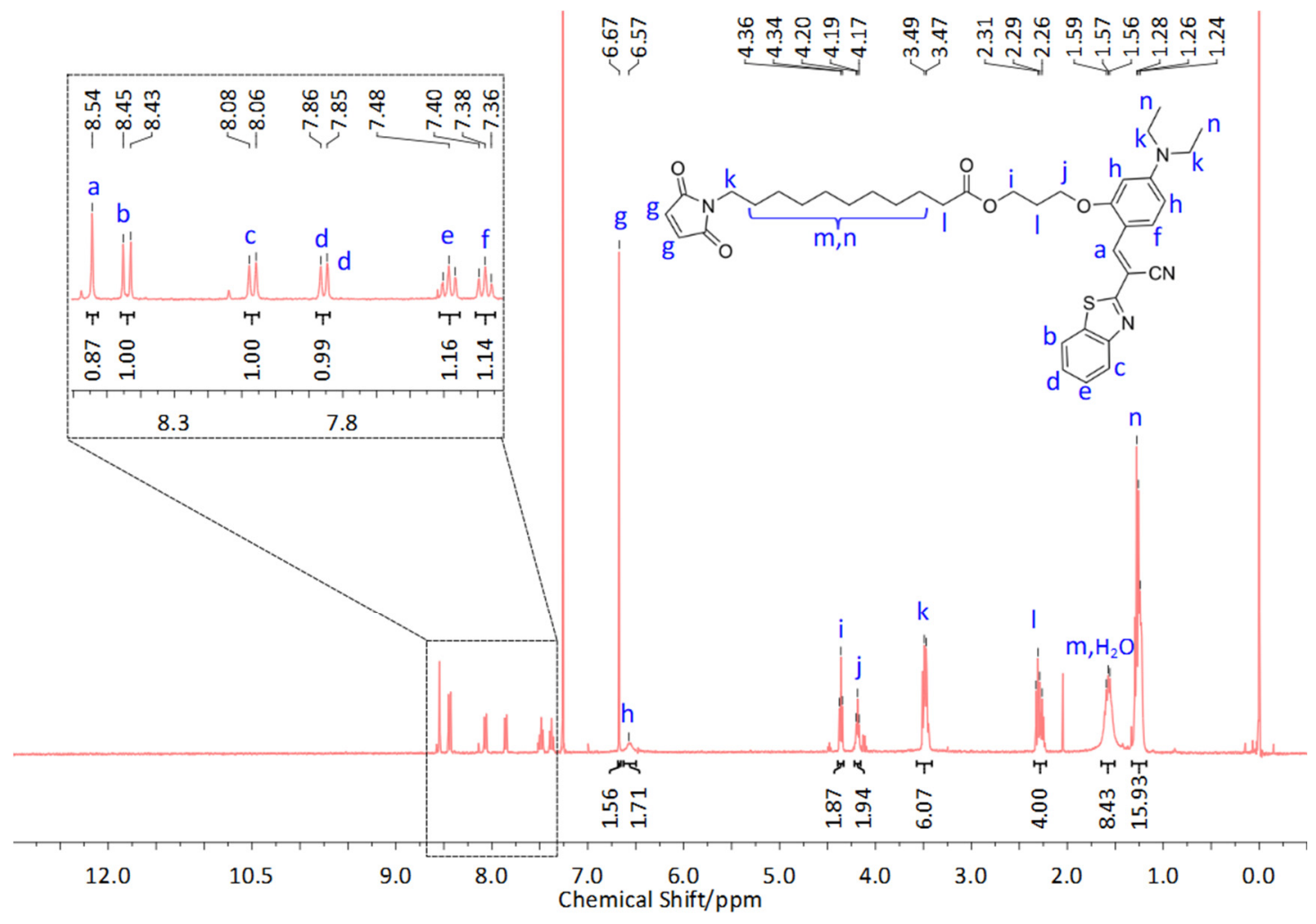

Figure S24. ${ }^{1} \mathrm{H}$ NMR spectrum of the molecular precursor (MCV) in $\mathrm{CDCl}_{3}$. 


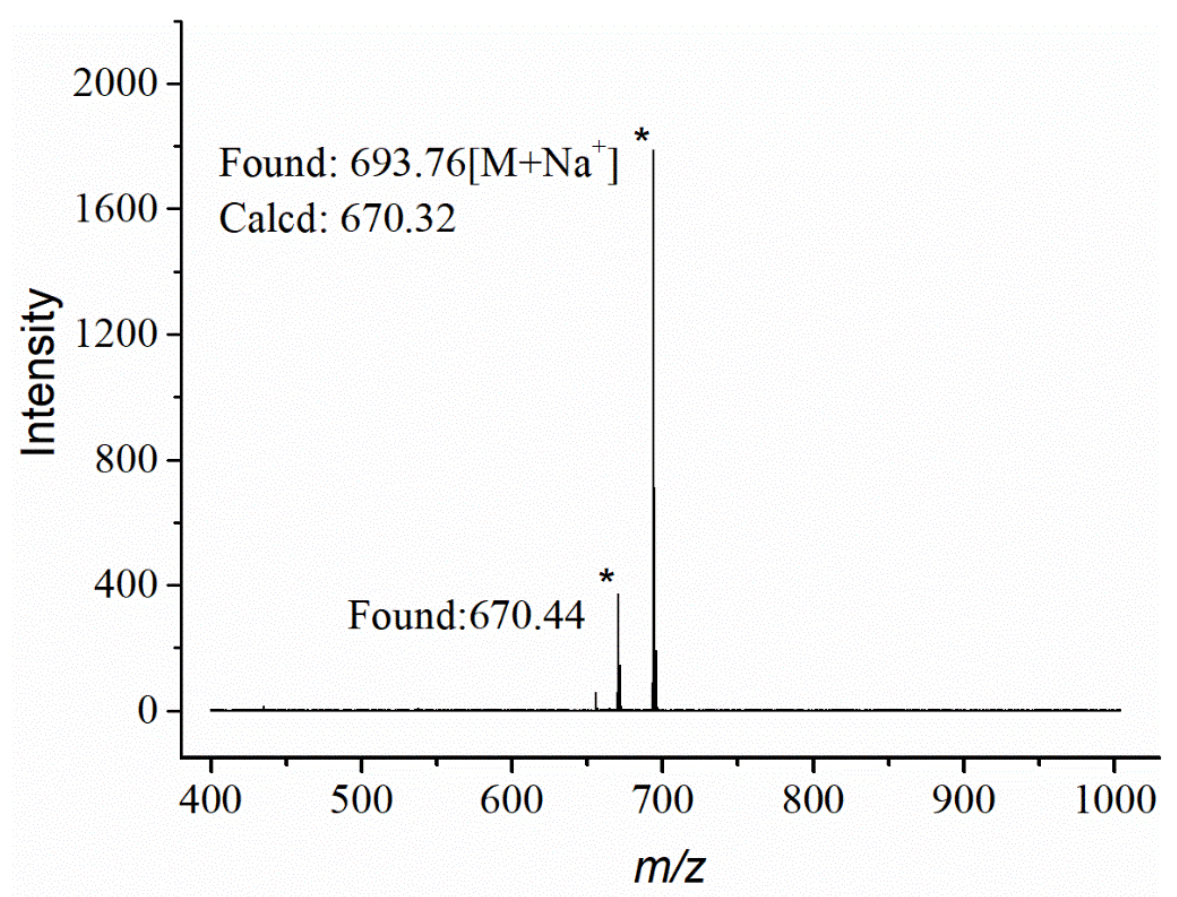

Figure S25. MALDI-TOF mass spectrum of the molecular precursor (MCV).

\section{Reference}

(1) Ito, S.; Tanaka, Y.; Kakehi, A.; Kondo, K. Bull. Chem. Soc. Jpn. 1976, 49, 1920.

(2) Song, W.; Wang, Y.; Qu, J.; Madden, M. M.; Lin, Q. Angew. Chem. Int. Ed. 2008, $47,2832-2835$.

(3) Glassner, M.; Oehlenschlaeger, K. K.; Welle, A.; Bruns, M.; Barner-Kowollik, C. Chem. Commun. 2013, 49, 633-635.

(4) Han, G.; Kim, D.; Park, Y.; Bouffard, J.; Kim, Y. Angew. Chem. Int. Edit. 2015, 54, 3912-3916.

(5) Zhang, L. L.; Geng, Y.; Jin, Z. J. Org. Chem. 2016, 81, 3542-3552.

(6) Liu, G. Y.; Zhuang, W. H.; Chen, X. B.; Yin, A. L.; Nie, Y.; Wang, Y. B. Polymer 2016, 100, 45-55.

(7) Wang, Y. Z.; Rivera Vera, C. I.; Lin, Q. Org. Lett. 2007, 9, 4155-4158. 